\title{
Light forces the pace: optical manipulation for biophotonics
}

\author{
David James Stevenson \\ University of St Andrews \\ Scottish Universities Physics Alliance \\ School of Physics and Astronomy \\ North Haugh, Fife, KY16 9SS United Kingdom \\ and \\ University of St Andrews \\ School of Biology \\ North Haugh, Fife, KY16 9TS United Kingdom
}

\section{Frank Gunn-Moore}

University of St Andrews

School of Biology

North Haugh, Fife, KY16 9TS United Kingdom

\section{Kishan Dholakia}

University of St Andrews

Scottish Universities Physics Alliance

School of Physics and Astronomy

North Haugh, Fife, KY16 9SS United Kingdom

\begin{abstract}
The biomedical sciences have benefited immensely from photonics technologies in the last 50 years. This includes the application of minute forces that enable the trapping and manipulation of cells and single molecules. In terms of the area of biophotonics, optical manipulation has made a seminal contribution to our understanding of the dynamics of single molecules and the microrheology of cells. Here we present a review of optical manipulation, emphasizing its impact on the areas of single-molecule studies and single-cell biology, and indicating some of the key experiments in the fields. (C) 2010 Society of Photo-Optical Instrumentation Engineers. [DOI: 10.1117/1.3475958]
\end{abstract}

Keywords: optical tweezers; single-molecule studies; molecular motors; single-cell studies; microfluidics; Raman spectroscopy; optical sorting; optical stretching.

Paper 10094VSSR received Feb. 24, 2010; revised manuscript received May 27, 2010; accepted for publication Jun. 9, 2010; published online Aug. 17, 2010.

\section{Introduction}

The year 2010 marks the $50^{\text {th }}$ anniversary of the laser. Among the very wide impact lasers have had in modern science, one of the most exciting and significant advances in the last four decades has been in the application of optical forces across all the sciences. The reader is likely to be familiar with the uses of light for imaging and spectroscopy, but the concept of light exerting a force may perhaps appear rather less mainstream or, indeed, applicable. However, at the mesoscopic scale (nanometer to micrometer), the mechanical effects of optical fields have made a significant impact that is not restricted to biological material but encompasses colloidal samples and even atoms and molecules. Historically, we have to first look to Kepler, 400 years ago, for the origins of such optical forces. He published his conclusions regarding the tails of comets pointing away from the sun at all points on their highly eccentric orbits in De cometis libelli tres (1619). ${ }^{1}$ To explain his observations, Kepler conceived the notion of some form of solar light pressure-a revolutionary proposition signifying the basis for the first ideas that light could exert a force. At the turn of the 20th century, these concepts became more apparent with the experimental studies of radiation pressure. ${ }^{2,3}$

In this review, we are more concerned with microscopic entities rather than astronomical ones, and it was the advent of the laser in 1960 that opened up a range of new opportunities to manipulate mesoscopic particles. Ashkin ${ }^{4}$ was the key pioneer of this new field and performed the seminal experiments with various colleagues. His original experiments were using

Address all correspondence to: David J. Stevenson, University of St Andrews, Scottish Universities Physics Alliance, School of Physics and Astronomy, North Haugh, Fife, KY16 9SS, United Kingdom. Tel: 44-781-119-2687. E-mail: ds50@st-andrews.ac.uk micron-sized spheres with a visible gas (argon ion) laser. In a liquid sample medium containing a dispersion of microspheres, he directed a single horizontally propagating laser beam and saw the microspheres align with the beam propagation axis. These microspheres were seen to be pushed along the laser beam direction due to the radiation pressure of the beam. This was the first observation of optical guiding: the transport of particles along the bright center of a light field. The optical forces can be explained as being due to the gradient of the light field drawing the object into the beam axis and a radiation pressure (scattering) component propelling the particle along the beam propagation axis. These ideas are valid for particles of higher refractive index than their surroundings. Ashkin added a second counterpropagating light beam (of equivalent optical power) creating a radiation pressure force in the opposite direction. Under these conditions, the particle was held at rest between the two laser beams and this constituted the first optical trap using this dual-beam trap geometry. ${ }^{4}$ The use of single-mode optical fibers 20 years after this first observation made this dual-beam trap system more practical. ${ }^{5}$ Such a counterpropagating beam trap has had relevance to studies in biophotonics, and we shall return to it in due course: in particular, it has assisted in studies of the cell stretcher, ${ }^{6,7}$ Raman analysis, ${ }^{8}$ and it is noted for its compatibility with microfluidic environments. The interest in this trap stems largely from the absence of high numerical aperture optics, with the trap being formed between two weakly focused beams. This produces a large confinement volume with the capability to distribute the optical force over a substantial region, allowing, for example, the trapping of large cells as well as providing the basis for studies in longitudinal binding of microscopic colloidal particles. ${ }^{89}$ The lower power density

1083-3668/2010/15(4)/041503/21/\$25.00 @ 2010 SPIE 
of these traps also means potentially increased viabilities of held biological material.

Following these dual counterpropagating beam studies, Ashkin and Dziedzic ${ }^{10}$ also investigated the stability of trapping hollow and glass spheres with optical beams, balancing optical forces against gravity. In the case of hollow spheres, annular light fields were used ${ }^{11,12}$ because they have a refractive index lower than their surroundings and thus are repelled from bright intensity regions. The year 1986 was a key year in the development of optical manipulation: Ashkin et al. demonstrated particle confinement with the single-beam gradient trap (popularly known as optical tweezers). ${ }^{13}$ This is now established as the major method for applying optical forces to hold and manipulate microscopic particles, and as such optical tweezers have now been recognized as probably having the greatest impact in the field of optical micromanipulation and biophotonics.

From these early studies, optical manipulation has seen a consistent and ever-increasing impact across a variety of different fields. For example these very same gradient and scattering forces have been preeminent in studies of laser cooling and trapping of atoms. However, these fields will be outside the scope of this paper and we refer the interested reader to relevant literature. ${ }^{14-16}$ Although there are also several recent review papers in the general field of trapping pertaining to mesoscopic particles and cells, ${ }^{17-22}$ the present overview focuses on the applications of optical manipulation in biophotonics, and in particular, cell biology and single-molecule biophysics. This paper is organized as follows: First, we describe the theoretical basis for single-beam optical trapping, including a discussion of how the forces may be understood to operate at different size scales. We then progress to describe experiments related to cell biology and single molecule studies. Our aim is not to give a comprehensive list of the experiments in these areas but rather highlight some of the major studies with a particular emphasis on their biological importance. We conclude with a discussion on the future applications of optical manipulation in the life sciences.

\subsection{Theoretical Basis for Single-Beam Optical Trapping}

Optical manipulation is a term we use to encompass the application of optical forces to move, guide, and trap (in 2-D and 3-D) mesoscopic objects. Optical tweezers-single-beam 3-D trapping - is the method we describe here in great detail, given its importance for the field of biophotonics. Optical tweezers rely on a highly inhomogeneous spatial field distribution of the beam. This causes a small but significant optical force (approximately a piconewton) to act upon a dielectric or biological particle (of higher refractive index than its surroundings) near the focus and is the case we deal with here. This attracts the particle toward the highest intensity part of the field through what is commonly termed the gradient (dipole) force. A stable equilibrium is attained where any opposing force originating from light scattering becomes equal in magnitude. Consequently, such a particle can be trapped in a stable equilibrium when it is very close to the beam focus. For further details of the theoretical basis of optical trapping, we refer the reader elsewhere. ${ }^{19}$
Notably, any thermal forces can be detrimental compared to the optical forces. Such thermal forces typically originate from temperature gradients in the medium surrounding the particles caused by the absorption of radiation, creating an effect known as photophoresis. ${ }^{23}$ Thus, optical trapping is typically restricted to the use of relatively transparent media and particles where any thermal effects are negligibleindeed, in the context of biophotonics, any residual heating can adversely affect cell viability and should be avoided. We shall discuss this later in the context of applications of trapping to cell biology.

The ratio between the dimension of the trapped particles (of radius $r$ ) and the wavelength of the trapping laser source (denoted by $\lambda$ ) determines how we mathematically treat the light-particle interaction. First, if we consider a dielectric particle whose radius is much smaller than the optical wavelength, the particle may be considered as an oscillating dipole, and the appropriate mathematical formulation to be used is the Rayleigh limit. ${ }^{13,24,25}$ Second, for cases where the particle size significantly exceeds the trapping laser wavelength, the geometric optics or ray optics regime suffices to describe the phenomena of optical forces that result from refraction and reflection. $^{26,27}$ We will look at both the Rayleigh and ray optics limits in a little more detail.

\subsection{Rayleigh Regime}

To implement an optical trap entails the use of a highly intense and strongly focused beam. The trap occurs from a balance between the gradient force pulling the particle of a higher refractive index than its surroundings to the most intense region of the optical field (i.e., the beam focus), and the repulsive scattering force, which pushes the particle away from the beam focus. If we consider the dipole approximation, then the force on a particle is given by the Lorentz force

$$
\mathbf{F}=(\mathbf{p} \cdot \nabla) \mathbf{E}+\dot{\mathbf{p}} \times \mathbf{B},
$$

where $\mathbf{E}$ and $\mathbf{B}$ denote the electric and magnetic fields of the radiation, $\mathbf{p}$ is the particle's induced dipole moment, and $\dot{\mathbf{p}}$ is its time derivative. The first term in Eq. (1) denotes the interaction of the induced dipole with the light field and yields what is commonly termed the gradient force. The second term in Eq. (1) represents a force that fluctuates with the laser frequency. Thus, in terms of the dynamics of optical trapping this term has no relevance.

The total Lorentz force, experienced by a particle in a medium of refractive index $n_{m}$, naturally includes both a gradient and scattering force. If we consider a linear response to the (plane-wave) electric field and that $\mathbf{p}=\alpha \mathbf{E}$, where $\alpha$ is the polarizability, then the total optical force may be written as ${ }^{13,24,25}$

$$
\mathbf{F}=-\frac{n_{m}}{2} \alpha \boldsymbol{\nabla}\left(E^{2}\right)+\frac{16 \pi^{4} \alpha^{2}|E|^{2}}{3 \varepsilon_{0} n_{m} \lambda^{4}} \hat{\mathbf{k}},
$$

where $\lambda$ is the laser wavelength, $\mathbf{k}$ is the radiation wave vector, and $\varepsilon_{0}$ the vacuum permittivity. The effective particle polarizability $\alpha$ for a homogeneous dielectric particle is given by 


$$
\alpha=4 \pi \varepsilon_{0} n_{m}^{2} r^{3}\left(\frac{m^{2}-1}{m^{2}+2}\right) .
$$

$m=n_{p} / n_{m}$ is the ratio of the refractive indices where $n_{p}$ is the refractive index of the trapped particle. A phenomenological correction to the polarizability expression (3) may be added in order to account for scattering and attenuation of the incident light field. ${ }^{25}$ We see that the polarizability is proportional to the particle volume: as the trapped particle's size reduces, the polarizability in Eq. (3) progressively evolves toward the dynamic molecular polarizability.

Optical trapping in the Rayleigh regime has been demonstrated in numerous experiments with dielectric and metal microparticles with sizes ranging from a few tens of nanometers upward. $^{28-31}$ It is interesting to comment on the exact role in optical trapping of the polarizability $\alpha$ and refractive index because these are issues of particular interest when considering metallic nanoparticles. In any material, the polarizability depends on the electronic properties and the mutability of the charge distribution under the influence of the electromagnetic field. Typically, the index of refraction is an effective medium concept based on a homogenization of the microscopic polarizability over the particle in question. This assumes that the constituent molecules or atoms of the material experience the equivalent environment that indeed is the same as the bulk counterpart.

For dielectric nanoparticles, this approach is valid because the local polarizability originates from bound electrons. Generally, the gradient force is proportional to the particle volume and, for this reason, it is very difficult to trap inert dielectric nanometer-sized objects. Metallic particles, though, differ in several respects, as per unit volume they possess a much larger inherent polarizability. To model the optical forces exerted on metal nanoparticles accurately, dispersion needs to be considered. Furthermore, the behavior of the conduction electrons alters when these electrons are in close proximity to the metal surface. If the particle dimensions are smaller than the corresponding skin depth, then the incident field interacts with the whole particle volume, resulting in a linear dependence between force and volume, resulting in the particle being essentially transparent. However, for particle sizes greater than the skin depth, the field interacts only with a thin outer layer and the force varies as the square of the diameter. ${ }^{31-35}$ Such considerations are of importance for the biomedical community because metallic nanoparticles have come to prominence in a number of studies for their ability to enhance of Raman signals, as photothermal agents in the selective destruction of cancer cells, as highly sensitive molecular diagnostic indicators, ${ }^{36}$ as well their ability to act as handles using optical forces. ${ }^{29}$

\subsection{Ray Optics Regime}

Optical tweezers are also able to trap larger particles, on the micron scale. At this scale, the particle is sufficiently large for the propagation of light through the trapped sphere to be determined through the principles of simple ray optics. Calculations based on the reflection and refraction of light on a model sphere may be used to determine the optical forces. Figure 1 shows a qualitative representation of a dielectric particle trapped by a single optical beam. ${ }^{26}$ We may decompose the

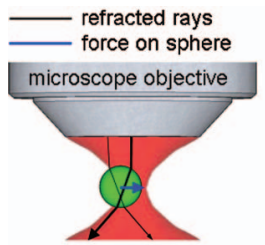

(a)

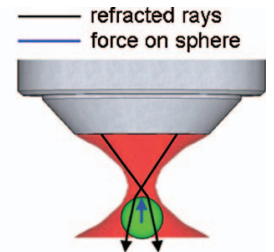

(b)

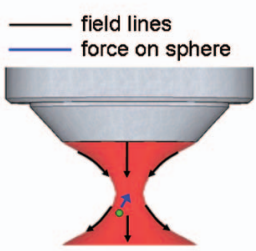

(c)

Fig. 1 Qualitative geometrical view of trapping, applicable to large spherical particles. A selected pair of rays demonstrate that the refraction from the trapping beam results in force attracting the particle toward the beam focus. For simplicity, we ignore the reflections because they do not play an important role in the final force balance. For a more detailed explanation, the reader is referred to Ref. 13 and 26. (a) Shows the lateral restoring force upon the sphere towards the beam focus. (b) Shows the axial restoring force acting upon a sphere that is axially displaced downstream from the focal plane. Were the bead to be located upstream of the focal plane, it can be simply shown that the restoring force would point downward. (c) Outside the scope of geometrical optics, for Rayleigh particles it is the particle's polarizability, and not its transparency, that leads to trapping. The particle is aligned to the center of the trap because the electric field component of the light generates an induced dipole in the sphere, which will align in the highest region of the field gradient (in the static field approximation).

entire beam into individual rays, each with an appropriate intensity, direction, and polarization, propagating in straight lines within a medium of refractive index $n$. The individual rays can change direction when each reflects or refracts, and may change polarization at dielectric interfaces in a manner determined by the Fresnel equations. The refraction or reflection of a light ray at the particle-medium interface results in a transfer of momentum from the trapping laser to the particle.

From this description, we can calculate the forces due to individual rays of power $P$ and the ray incident angle on the surface of the sphere surface. The quantities $R$ and $T$ denote the Fresnel reflection and transmission coefficients of the surface, and we find that the total force exerted on a particle is the sum of the contributions due to the reflected rays of power $P R$, and an infinite number of emergent refracted rays, of successively decreasing power, $P T^{2} R^{m}$, where $m=0,1,2, \ldots$, leading to the overall force as ${ }^{27}$

$$
|\mathbf{F}|=|\mathbf{Q}| \frac{n_{m} P}{c}
$$

Here, $n_{m}$ is the refractive index of the medium and the trapping efficiency $|\mathbf{Q}|$ is dependent on the Fresnel coefficients $R$ and $T$, in essence giving us a measure of how much momentum has been transferred to the sphere from the light. For a more thorough analysis, see Ashkin. ${ }^{26}$ 


\subsection{Mie and Lorenz-Mie Regime}

The Rayleigh and Mie (ray optics) regimes are important, but the majority of experiments employ trapped particles whose sizes are actually comparable to the trapping laser wavelength. In this case, classical electrodynamics is required and the net optical force exerted on an arbitrary object may be calculated by the Maxwell stress tensor $\stackrel{\leftrightarrow}{\mathbf{T}}(\mathbf{r}, t)$ approach $^{37}$

$$
\langle\mathbf{F}\rangle=\oint_{S}\langle\stackrel{\leftrightarrow}{\mathbf{T}}(\mathbf{r}, t)\rangle \mathbf{n}(\mathbf{r}) d a,
$$

where the angular brackets denote a time average and the integral over $S$ signifies a closed surface enclosing the particle, with $\mathbf{n}(\mathbf{r})$ defining the outward unit vector normal to the integration surface. The optical force expressed in these terms is of general validity for an arbitrary (albeit rigid) body within the surface. It is entirely determined by the electric and magnetic fields at the surface. For deformable objects, additional electro- and magnetostrictive forces must be included. The fields used for the calculation of force using Eq. (5) are selfconsistent fields (i.e., super-positions of the incident and scattered fields).

Detailed calculations of this approach can be found in work by Barton ${ }^{38}$ and Barton et al. ${ }^{39}$ The stress tensor can be calculated for a sphere with the help of Mie theory, considering all fields inside and outside of the sphere to be continuous at the surface. An alternative approach to the Maxwell stress tensor has been described by Rohrbach ${ }^{40}$ and Rohrbach and Stelzer. ${ }^{41}$ One advantage of this approach is that the particles can take on an arbitrary shape and the interaction can be determined for arbitrary modes of the electromagnetic field.

Having an appreciation now of the theoretical platforms used to explain the optical forces and confinement of mesoscopic particles, we briefly comment on how such optical gradients are created, in practice, to produce single-beam optical tweezers. In the majority of systems, high numerical aperture microscope objectives with high magnification are used. Typically, a numerical aperture of $>1.2$ is used and, in turn, this produces the necessary strong lateral and axial gradients of the light field, yielding strong gradient forces essential for 3-D optical trapping. The choice of laser is also important and will be discussed later in this paper. For full flexibility in maneuvering the trapped object, we need to effectively steer the beam across the sample plane without it ever drifting away from the back "entrance" aperture of our objective lens, and this is achieved using optical conjugates. A more detailed description of the assembly and implementation of single-beam optical tweezers can be found elsewhere. ${ }^{42,43}$ It is also possible to develop a homebuilt system by adapting commercially available microscopes. ${ }^{44}$ We will now discuss why optical traps have been so important and how they have been used in biophotonics.

\section{Traps for Precision Measurements}

Optical tweezers are undoubtedly the corner stone of many biophysics experiments. A typical molecular level experiment measures the position of a microsphere bound to a single molecule, as the single molecule performs a function or interaction with another molecule. Example molecular models that will be explored in more detail later include the movement of kinesin $^{45}$ or dynein ${ }^{46}$ along microtubule tracks, myosin motion along actin tracks, ${ }^{47}$ viral porter motor deoxyribonucleic acid (DNA) packaging, ${ }^{48}$ ribonucleic acid (RNA) polymerase along DNA during transcription, ${ }^{49}$ ribosomes along RNA during translation, ${ }^{50}$ and proteins during mechanical denaturation. ${ }^{51}$ Cells can also be subject to precision measurements using optical traps.

However, it is first important to understand how such a trap is converted into a true measurement apparatus, as this has been the key to its widespread use in biophotonics. Although we can invoke the models used above to understand the forces and trapping of a variety of objects, this does not directly show us how we may make an actual measurement of force or displacement. The major reason that this field has advanced is that an optically trapped particle in a buffer medium is an excellent example of an "ideal" overdamped oscillator with a frequency roll-off of $\sim 1 \mathrm{kHz}$. The buffer medium provides a natural damping to the motion of the particle and assists in its localization. Although the trapped microparticle is confined within a diffraction-limited laser beam, the particle's movements are confined to a much smaller volume than the beam diameter, and using modern positional analysis apparatus (e.g., a quadrant photodiode or fast cameras), the center of gravity of the particle may be tracked and established with nanometric accuracy.

The tightly focused trapping light beam creates a parabolic potential energy well for the trapped object. This parabolic nature of the potential implies that for small displacements away from trap center, the force is directly proportional to displacement, thus creating a potentially simple but powerful way of converting any displacements observed into measurements of force. The trapped object is akin to a small spring obeying Hooke's law. By imaging the trapped object on a position-sensitive detector, we can calibrate the particle displacement and thus record the trap stiffness. The Brownian dynamics and their calibration is the technique of choice for absolute measurements. Here, either the bead position is recorded, temporal correlation measurements made, or one obtains the power spectrum of the bead locations, which can show a characteristic roll-off frequency. The accurate determination of the position of the trapped object is achieved either via a quadrant photodiode detector (with a very high bandwidth of typically $10 \mathrm{kHz}$ ) or a fast camera. The center-ofgravity position of the particle is ascertained with appropriate sampling and can readily result in knowledge of the particle position to nanometer precision. The fluctuations of the trapped particle can be described by the Einstein-OrnsteinUhlenbeck theory of Brownian motion. This leads to a Lorentzian power spectrum from which the trap strength may be derived. For more details, we refer the reader elsewhere. ${ }^{52}$

In single-molecule studies, rather than trap a molecule such as DNA directly (which would be hard due to its extremely small size and thus small polarizability), a micro- or nanosphere is chemically functionalized and attached to the molecule of interest. Attachment chemistries include biotin-streptavidin, ${ }^{53}$ recombinant epitopes, such as his tags ${ }^{54}$ or immunological tags. ${ }^{55}$ A variety of experimental configurations are then employed depending on the molecule of interest and the type of forces that molecule generates. We briefly 
Table 1 Popular configurations used in single-molecule studies. In each configuration, forces are measured on the terminal microsphere using optical tweezers. An anchor can be where the molecule is bound to either a coverslip, a microsphere that is held firmly by the suction of an extruded glass capillary tube, or another optical trap. Table inspired by Perkins. ${ }^{56}$

\begin{tabular}{|c|c|c|}
\hline Name & Configuration & Example investigations \\
\hline Tug-of-war & anchor-protein-nucleic acid-microsphere & $\begin{array}{l}\text { RNA polymerase }{ }^{49,57,61} \\
\text { (e.g., Fig. 4), helicase, } \\
\text { portal motor }{ }^{48,59} \text { (e.g., }^{\text {e. }} \\
\text { Fig. 3), exonuclease }\end{array}$ \\
\hline Conversion & anchor-dsDNA-polymerase-ssDNA-microsphere & DNA polymerase ${ }^{63}$ \\
\hline Unzipping & anchor-ssDNA--dsDNA-ssDNA-microsphere & $\begin{array}{l}\text { DNA restriction enzymes, } \\
\text { helicase }^{65}\end{array}$ \\
\hline Popping & anchor-_enzyme bound dsDNA)_-microsphere & $\begin{array}{l}\text { Nucleosome } \\
\text { restriction enzymes }\end{array}$ \\
\hline $\begin{array}{l}\text { Fluorescent } \\
\text { tracking }\end{array}$ & anchor-_(fluorescent DNA)—enzyme & helicase/nuclease 69 \\
\hline
\end{tabular}

highlight some of the techniques available, but the reader is referred to a thorough review in Ref. 56.

When the force of an enzyme or molecular motor acting on a DNA molecule is to be measured, such as RNA polymerase for example, one configuration that may be used is called the tug of war (Table 1). Here, an anchored protein generates tension on a nucleic acid molecule during the protein's enzymatic activity, and the resulting force acting on a microsphere is assessed using optical tweezers. Proteins investigated in this way have included RNA polymerase,${ }^{57}$ helicase, ${ }^{58}$ portal motor, ${ }^{59}$ and exonuclease. ${ }^{60}$

Another configuration is called the conversion assay, where the forces acting on DNA during the enzymatic conversion of dsDNA and ssDNA may be monitored. The conversion assay measures the ratio of dsDNA/ssDNA over time and therefore gives the efficiency of the enzyme such as DNA polymerase. $^{63}$

The unraveling of a dsDNA strand into its two singlestranded components is often called an unzipping assay. During unzipping by force, bound enzymes (such as restriction enzymes) can be observed as an increase in tension during the unzipping process and their positions and equilibrium association constants $\left(K_{\mathrm{a}}\right)$ may deduced. ${ }^{64}$ Unzipping experiments have also investigated DNA unwinding by helicases. ${ }^{65}$

DNA in vivo is heavily looped by interaction with packaging proteins, such as histones. As tension is increased during the unravelling of a dsDNA strand that has been wound around a large protein core, such as the histones contained in a nucleosome, a characteristic popping occurs during force measurements. ${ }^{66}$ This allows the direct measurement of the extent of protein-DNA binding. This type of popping signal is also found in proteins that bind to DNA in two different, distant places, forming a DNA loop. One such protein is the ubiquitous restriction endonuclease, some varieties of which require two binding sites for cleavage to occur. ${ }^{68}$

Some single molecule experiments use optical tweezers, not as a force measuring device, but rather as a tool to micromanipulate single molecules into a different reagent stream such that a chemical reaction may be fluorescently tracked. Bianco et al. ${ }^{69}$ did just such an experiment in their investigation of single-RECbcd enzymes. RECbcd is a helicase and a nuclease; it both unwinds dsDNA and chops it into pieces as it moves along the length of the DNA. The authors optically tweezed a microsphere containing a bound DNA moleculethe DNA was stained fluorescently. At the distal end of the DNA molecule, an inactive RECbcd was bound. When the authors tweezed the assembly into a different reagent to activate the RECbcd molecule, they could observe (by fluorescence) in real time the shortening of the DNA molecule as the RECbcd degraded it. ${ }^{69}$ Handa et al. performed a similar investigation but monitored the displacement of a fluorescent nanoparticle bound to the RECbcd, rather than measuring the diminishing fluorescence of the degraded DNA. ${ }^{70}$

The above fluorescent tracking configurations did not overlay a tweezing beam and a fluorescent probe beam. Although it is possible to monitor fluorescence of a single molecule during its micromanipulation, this is technically challenging. Not only can the fluorophore of interest photobleach rapidly in the tweezing beam, the fluorescent signal can be as much as 15 orders of magnitude lower than the photon flux of the tweezers. ${ }^{71}$ One technique to limit this incompatibility is to modulate the tweezing and fluorescence excitation sources. This can lead to a 20-fold improvement in fluorescence longevity without a reduction in sensitivity or trapping stiffness. $^{72}$

Trapping and tracking the microsphere thus can indirectly give valuable information on the molecular dynamics that occur as the molecule performs its biological function. The crucial step here is to create a useable force transducer that can be calibrated, and this is achieved by use of a fast positiondetection system.

A major area of application for this technique is microrheology, where optical traps can aid the measurement of the viscoelastic properties of polymers or macromolecules. In particular, optical tweezers can exert very small forces and the resulting real-time dynamics of macromolecules can be re- 
corded with excellent temporal resolution. Optical tweezers, like diffusing wave spectroscopy, are linear in measurements up to megahertz frequencies but, unlike diffusing wave spectroscopy, optical tweezers do not rely on ensemble measurements. In comparison, large-scale rheological devices are limited to $100 \mathrm{~Hz}$. Atomic force microscopy (AFM) and magnetic tweezers can also perform single molecule microrheology. Of these, there is no minimum size limit on the length of molecules able to be measured by AFM; smallersized molecules in tweezer systems result in the unwanted effect of two beads simultaneously hopping into the trap. Optical tweezers do offer an advantage of multiple trap sites (unlike magnetic tweezers) and the ability to raise the sample a high-enough distance from the bottom of the sample chamber well to remove the hydrodynamic influence of the boundary. The interested reader is referred to an excellent review comparing these various techniques. ${ }^{73}$

\section{Studies on the Dynamics of Single Molecules}

On the basis of these methods of calibration described above, optical tweezers have found numerous applications in singlemolecule studies. Why are such small forces and distances useful to exert and measure? Indeed, optical tweezers are unable to sever covalent bonds (typically, forces of nanonewtons are needed); however, protein-protein interactions and the forces produced by many rotary and linear molecular motors (e.g., actin-myosin, kinesin motion on microtubules) fall within the $1-50 \mathrm{pN}$ range, making the use of optical tweezers ideal for such studies. The forces exerted are thus at the piconewton scale, and thus, this technology complements other techniques, such as magnetic tweezers and AFM, ${ }^{74}$ where the forces are typically larger. It is worth remarking that until the advent of single-beam tweezers, much of the knowledge of single-molecule behavior was derived from ensemble studies.

We present some examples of single-molecule studies that have been enabled by optical tweezers. It is important to note that the optical trapping geometry has to be tailored to the specific study. More comprehensive reviews and detailed aspects of single-molecule experiments with optical traps may be found elsewhere. ${ }^{20,56,75,76}$

\subsection{Molecular Motors}

Our understanding of the workings of both linear motors, such as kinesin, myosin, and dynein, and rotary motors, such as bacterial flagella, have without a doubt been improved by the use of optical traps. They have provided the means for the analysis of molecular motion, step sizes, forces, and crucially how these mechanical movements are related to the underlying biochemistry. Some of the first investigations into mechanical enzymes and molecular motors with optical tweezers were performed by various laboratories in the late 1980s and early 1990s. Prior to this time, molecular motor analysis was limited to bulk biochemical data and the passive tracking of beads by video microscopy.

Molecular motors can either be processive or nonprocessive in nature. Examples of the former include DNA and RNA polymerase, kinesin, and myosin $\mathrm{V}$, and examples of the latter include myosin II. ${ }^{77}$ The key difference is the number of steps along the bound polymer the motor takes; processive motors bind to a polymer chain and take many steps, and nonprocessive motors bind and take only approximately one step before dissociating.

One of the most prominent examples of studies in this era is that of kinesin, ${ }^{45}$ which are microtubule associated mechanical enzymes responsible for anterograde vesicle transportation $^{78}$ and meiotic and mitotic spindle organization. ${ }^{79,80}$ Kinesin is a homodimeric molecule with a coiled stalk linked to two head domains. The hydrolysis of adenosine triphosphate (ATP) drives the movement of the two head domains along a microtubule (toward its plus end) in an asymmetric "hand-over-hand" motion. ${ }^{81}$ It is processive, moving $\sim 100$ steps before detaching. ${ }^{82}$

For processive motors such as kinesin, the single-bead optical tweezers assay is appropriate. Such an assay was demonstrated by Svoboda et al., ${ }^{83}$ where a microsphere coated with kinesin was placed onto microtubule tracks that were bound to a coverslip. The authors then integrated a dual-beam interferometer into an optical tweezers setup to analyze the beads movements. Prior to entering the objective, a Wollaston prism was used to split the laser light into two orthogonally polarized beams, which were then focused onto the specimen as diffraction-limited overlaid probe regions. The microsphere-bound kinesin, when entering or moving through the probe region, introduced a relative phase shift between the two beams which, after recombination via another Wollaston prism, manifested itself as ellipticity. This ellipticity was then measured by passing the beam through a quarter waveplate (to circularly polarize the light), a polarizing beamsplitter, and finally two photodetectors and a normalizing differential amplifier. The net result was a detection sensitivity of $<1 \mathrm{~nm}$, and after calibration, force measurements of the molecular motor could be made.

A number of key points relating biophysical movements to biochemical activity have been gleaned from the above study and subsequent work. Kinesin moves with $\sim 8 \mathrm{~nm}$ step sizes, ${ }^{83,84}$ which corresponds to the molecular spacing of tubulin dimers. Intriguingly, in reduced ATP conditions, the molecular motion after each such step stops, awaiting the addition of new energy in the form of ATP molecules from the surrounding buffer. Low concentrations of ATP cause single steps that are still $\sim 8 \mathrm{~nm}$; therefore, the hydrolysis of one ATP molecule is directly related to a single step..$^{81,83,85,86}$ Analysis of the timing of the kinesin movements implied that it remains completely bound during its activity, hence, confirming the "hand-over-hand" hypothesis ${ }^{83}$ (which was later shown to be asymmetric in nature ${ }^{81}$ ). Speeds ${ }^{87}$ of up to $800 \mathrm{~nm} / \mathrm{s}$ and forces of 5-7 pN for single kinesin molecules were measured. ${ }^{84,87,88}$ There are still many unanswered questions about the exact mechanism and dynamics of kinesin, and we refer elsewhere for a more in-depth discussion of many of the current issues. ${ }^{81}$

Myosin is another molecular motor, which consists of a long tail and two heads. Enzymatic digestion of myosin can divide it into smaller units, heavy meromyosin (HMM), which has a truncated tail but a fully functioning double head, and subfragment 1 (S1), which has a truncated tail and a single head. Its collective action along actin monomers drives muscle contraction, and in a sense, it can be considered as a nanoactuator. To explore the dynamic actin-myosin system, a 


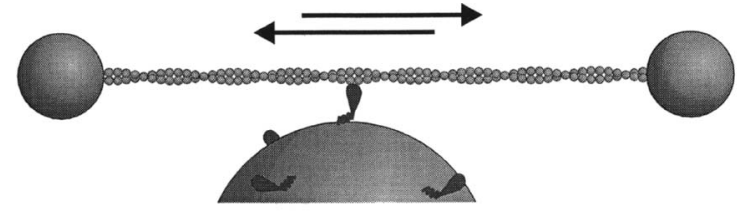

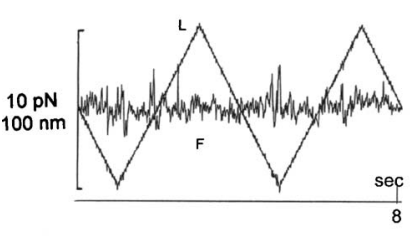

(a)

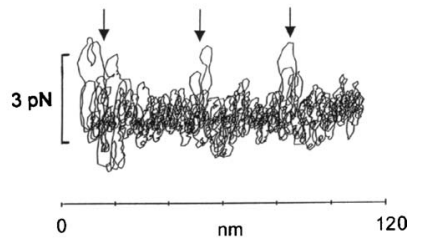

(b)
Fig. 2 The movement of a single S1 myosin head along an actin filament. See text for details. (This image was published in Ref. 91. (c) Elsevier.)

different experimental geometry was required from the previous kinesin studies. This is because nonprocessive motors such as myosin only bind briefly to their actin substrate before detaching. To measure this molecular motion, Finer et al. ${ }^{47}$ developed a novel assay in which an actin molecule was held between two beads and a third bead functionalized with myosin was brought into contact with the actin. Forces on the beads were then measured using a calibrated optical tweezers system. When the actin-myosin made contact, ATP in the buffer medium was hydrolyzed, resulting in the motion (a single "kick") of actin. One of the two trapped particles was imaged onto a quadrant photodiode to allow monitoring of these "kicks" over extended periods of time. Step sizes of $11 \mathrm{~nm}$ and forces of 3-6 pN were measured. ${ }^{47,89}$

Significant insight into the mechanics of myosin movement along actin monomers was gained by the subsequent experiments of Molloy et al. ${ }^{90,91}$ The authors demonstrated that single-myosin S1 heads acted as independent force generators when moving along actin filaments. Conceptually, unlike kinesin, which moves forward in an asymmetric "handover-hand" motion, a both double-headed HMM and a singleheaded S1 myosin fragment use a single "hand" or head to move along actin by repeatedly binding and releasing. Like the Finer study before it, the authors held an actin molecule suspended between two trapped $1.1 \mu \mathrm{m}$ diameter beads, and the filament was then placed near either an HMM (doubledheaded) or S1 (single-headed) functionalized bead that was attached to a coverslip. The position of one of the beads was accurately measured using a quadrant photodiode. Another observation by the same study is highlighted in Fig. 2. Here, the authors moved the two actin beads laterally in a sinusoidal motion and plotted length and force against time [Fig. 2(a)] and force against position [Fig. 2(b)]. This showed that the myosin S1 head attachment occurred at regular positions (arrows) $\sim 40 \mathrm{~nm}$ in distance from each other, corresponding to the periodic $38.5 \mathrm{~nm}$ twist in the actin structure.

Optical tweezers have also helped elucidate the biophysical characteristics of the related molecular motor, dynein. Dynein is a microtubule-associated molecular motor that drives the motion of the axoneme, the core of eukaryotic flagella or cilia. ${ }^{92}$ Like kinesin, dynein "walks" along microtubules and, in so doing, transports a variety of cytoplasmic vesicular
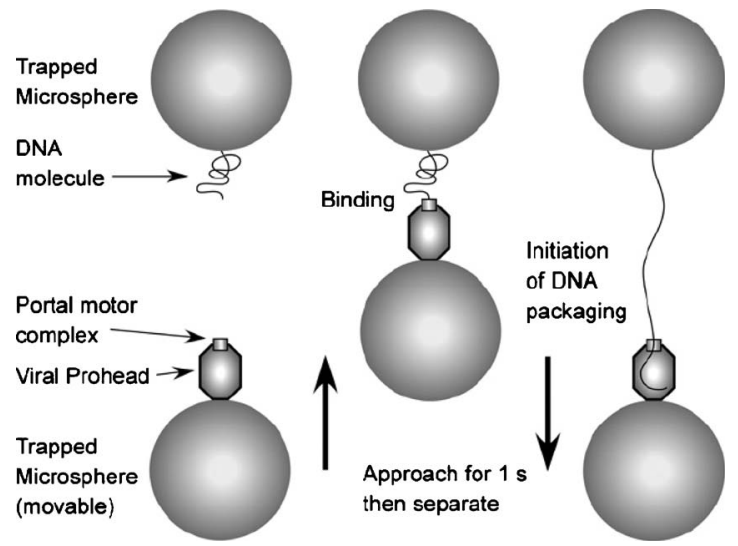

Fig. 3 Assessing the molecular motor, portal motor complex, by optical tweezers. A portal motor complex attached to a microsphere was manipulated via an acousto-optic deflector to a DNA bound bead for $\sim 1 \mathrm{~s}$ in order to initiate packaging. Once initiation commenced, forces on the DNA-bound sphere were measured by recording the deflection of the laser on the top sphere. (Reprinted from Ref. 48 (C) 2008, with permission from Elsevier.)

cargo, but a key difference is that kinesin walks toward the plus end of microtubules and dynein walks toward the minus end. It is also involved with spindle assembly, retrograde vesicle transportation, ${ }^{46}$ and chromosome segregation. Only a handful of articles have been written on the subject dynein force measurement by optical tweezers. Shingyoji et al. optically tweezed singlet microtubules via bead handles and overlaid them perpendicular to doublet microtubules; the authors subsequently measured the forces acting on the singlet arm by the connecting dynein molecules. ${ }^{93}$ To activate the dynein, they used UV flash photolysis to uncage ATP and, from these studies, they established that dynein exhibited a peak force of $\sim 6 \mathrm{pN}$. Hirakawa et al. ${ }^{94}$ later showed that processive movements of dynein are dependent on the ATP concentration; only low concentrations of ATP allow processive movement to occur. More recent studies demonstrated $8 \mathrm{~nm}$ step sizes for dynein, but these step sizes can vary between 4 and $24 \mathrm{~nm}$, depending on the load it is carrying. ${ }^{95}$

Other molecular motors have also been characterized with optical tweezers. During infection of bacteria, the viral bacteriophage $\phi 29$ packages DNA into precursor capsids via a molecular motor called a porter motor. Rickgauer et al. ${ }^{48}$ were able to measure the force and rate with which the motor assembly packaged dsDNA into the viral prohead (Fig. 3). Their experiment consisted of two tweezers, one of which was controlled by an acousto-optic deflector, and the other was static, and using this setup, the deflection forces were measured on the static trap using a position sensing detector. Essentially, this is the tug-of-war configuration discussed in Sec. 3 (Table 1). Using an improved protocol from the original 2001 study, ${ }^{59}$ the Rickgauer group attached a portal motor complex to one sphere, DNA to another, tweezed them together to initiate packaging, and monitored the resulting forces. The authors then showed that this motor has an average power of $\sim 700 \mathrm{zW}$ with a $30 \mathrm{pN}$ load and a $65 \mathrm{bp} / \mathrm{s}$ velocity. From these studies, there is now a growing interest in the use of genetically engineered variants of the $\phi 29$ motor, adapted to be lipid membrane bound, for a variety of biomedical and 
nanotechnological applications. ${ }^{96}$ It also furthers our understanding of bacteriophage, which has been proposed one day to be used therapeutically against multidrug resistant bacteria. $^{97}$

\subsection{Studies on Nucleic Acids}

\subsubsection{Measuring the transcription of DNA}

Many molecular-scale studies with optical traps have focused on exploring transcription. The transcription of DNA into an RNA molecule begins with the binding of a promoter region by RNA polymerase, ${ }^{98}$ followed by initiation, elongation, and finally termination. During binding, the closed DNA helix is melted and bent by RNA polymerase, resulting in an open conformation. ${ }^{99}$ Using optical tweezers in a tug-of-war configuration, early experiments showed that RNA polymerase exerted a force of $\sim 14 \mathrm{pN}$ during transcription. ${ }^{61}$ Force analysis of RNA polymerase during transcription of DNA to messenger RNA demonstrated that the step size of the RNA polymerase is equivalent to the distance of a single base pair or $0.37 \pm 0.06 \mathrm{~nm}$ (Fig. 4). ${ }^{49}$ This latter experiment was a major step forward, from a technological viewpoint, because it allowed angstrom-level position analysis. This advance was achieved by assembling an ultrastable trapping apparatus that included $(i)$ a dumbbell arrangement that obviates problems associate with stage drift, (ii) an all-optical passive force clamping arrangement, where one of the beads is held near the peak of the force extension curve [here, force and position are independent, Fig. 4(a), white arrow], and (iii) the use of helium in the beam path to reduce any laser beam drift.

\subsubsection{Detecting the cleavage of DNA by restriction enzymes}

Depending on its microenvironment, DNA can exist in various states of coil, spanning from uncoiled to supercoiled. As a result, its state affects the ability of a restriction enzyme to find and cleave its target sequence. Indeed, from in vitro studies, it is has been demonstrated that restriction enzymes find and cleave their targets more quickly in supercoiled than uncoiled DNA. Recently, direct evidence of this effect was demonstrated on single DNA molecules exposed to saturating concentrations of a restriction enzyme. ${ }^{62}$ By optically tweezing a linearized dsDNA molecule between two bead handles and then introducing an EcoRV restriction enzyme, these studies confirmed that the average time it took for cleavage to occur was longer when the molecule was stretched than when relaxed.

\subsubsection{Measuring the transporting of DNA through a nanopore}

It is possible to measure directly the force acted on a single DNA molecule as it is threaded through a nanopore due to electrophoresis. In an experiment described by Sischka et al., ${ }^{100}$ a single linear dsDNA molecule was tagged with a bead handle on one end and placed near the entrance of an $80 \mathrm{~nm}$ diameter pore previously milled on $20 \mathrm{~nm}$ thick $\mathrm{Si}_{3} \mathrm{~N}_{4}$ membrane (Fig. 5). On the application of a $100 \mathrm{mV}$ potential difference across the membrane, the charged dsDNA molecule threaded itself through the membrane pore and, as it did so, the force acted on the bead handle was measured to be $16 \pm 2.5 \mathrm{pN}$. With this technique, the position of the DNA

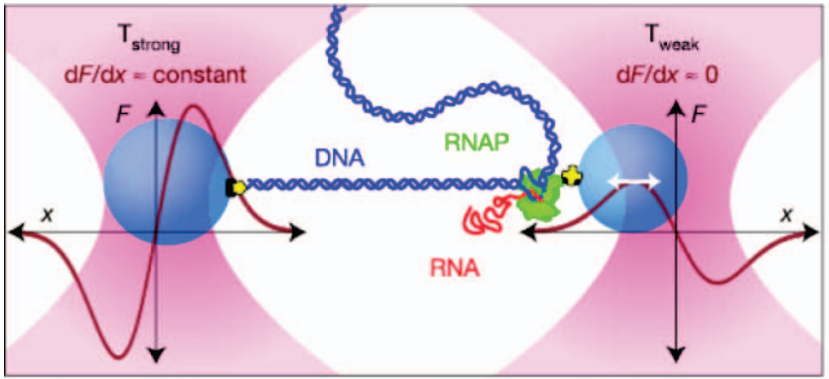

(a)

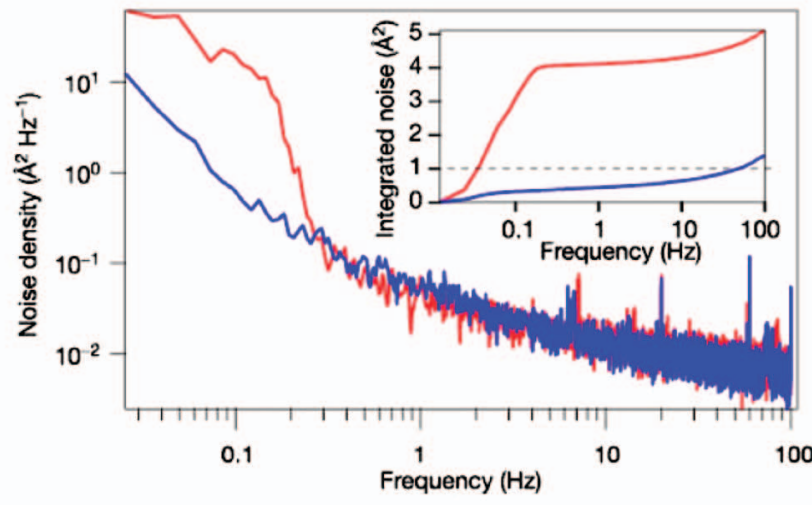

(b)

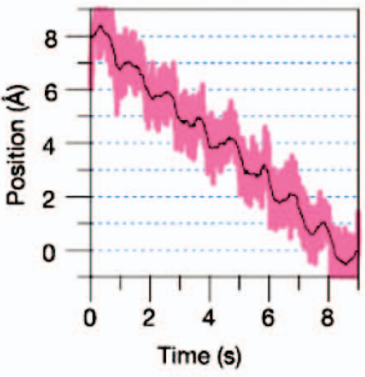

(c)

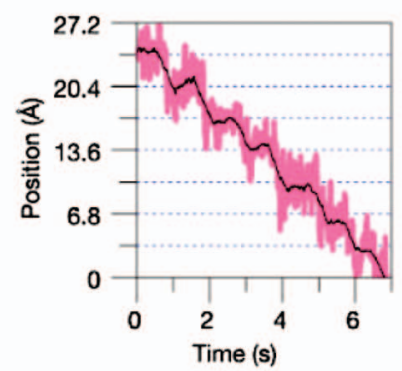

(d)
Fig. 4 A highly sensitive tug-of-war assay able to measure angstrom level position. (a) The tug-of-war assay showing a DNA molecule bound to a bead (left), which is held in a strong optical trap, coupled to an RNA polymerase-bound bead held in a weak optical trap, where displacement is measured as the RNA polymerase extends itself along the molecule. (b) Power spectrum for a bead held in air (red) or helium (blue), and an integrated noise spectra (inset) showing a tenfold reduction in power. (c) Steps resolved for a trapped bead moved at $1 \AA$ increments and (d) steps resolved for a bead-DNA-bead dumbell moved at $3.4 \AA$ steps. (Reprinted with permission from Macmillan Publishers Ltd., Nature, Ref. 49, (C) 2005.)

molecule within the pore-to-nanometer resolution is possible. ${ }^{101}$ The charge of individual DNA molecules can also be measured, which previously had to be taken from ensemble studies using electrophoresis. ${ }^{102}$ Finally, there is excellent potential with this experimentation to perform single-molecule DNA sequencing, haplotyping, and to investigate DNAprotein interactions. ${ }^{103}$

\subsubsection{Measuring the translation of RNA into protein}

Recent studies with optical traps have also observed, in real time, the first steps of a ribosome during translation. Wen et 


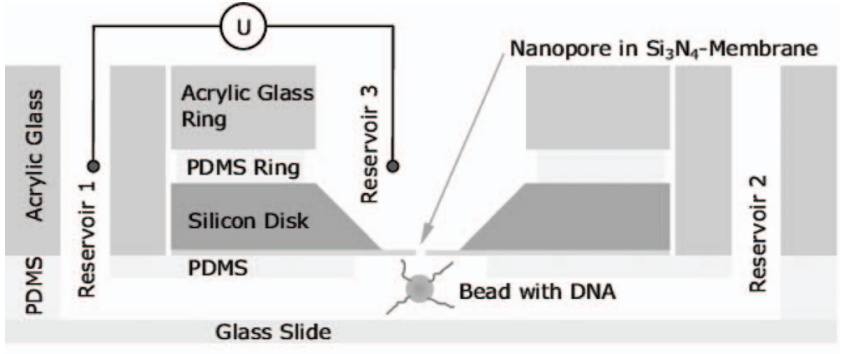

(a)

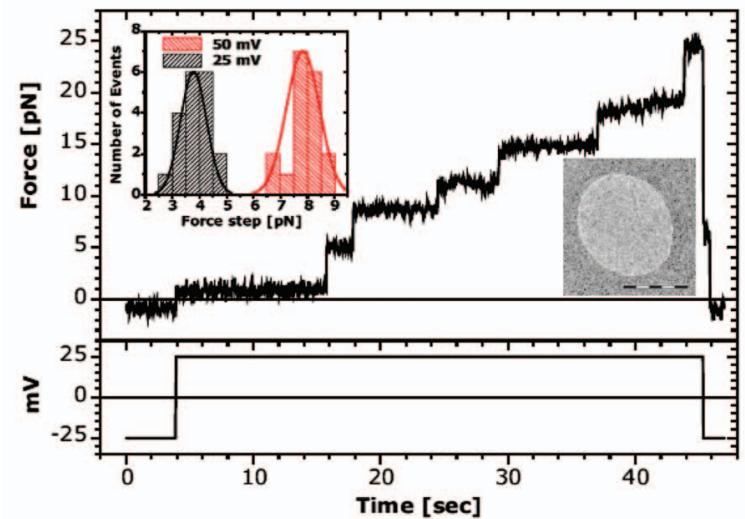

(b)

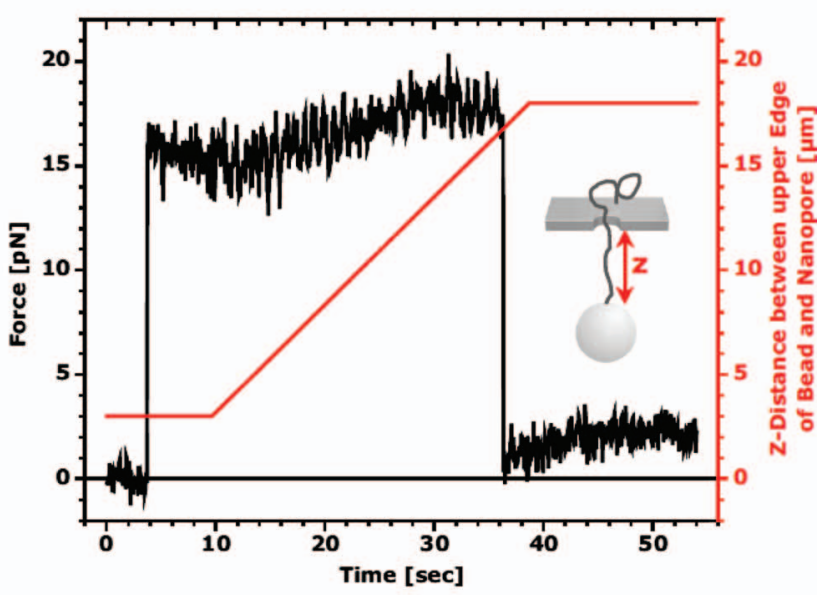

(c)

Fig. 5 (a) An $80 \mathrm{~nm}$ diameter nanopore was milled into a $20 \mathrm{~nm}$ thick $\mathrm{Si}_{3} \mathrm{~N}_{4}$ membrane, around which a custom fluid chamber was built. A microsphere functionalized with $\lambda$-DNA $(48,202$ base pairs, or $16.4 \mu \mathrm{m}$ in length) was placed in the bottom chamber, and an electrical potential difference of $\sim 25 \mathrm{mV}$ was applied across the membrane, resulting an almost instantaneous threading of the DNA through the pore. (b) Force measurements on the DNA during the threading were taken using optical tweezers. Each additional DNA molecule entering the pore generates an individual force step. (c) Active pulling of the DNA molecule out of the nanopore. (Reprinted with permission from Ref. 100. (c) 2008, American Institute of Physics.)

al. ${ }^{50}$ followed the movement of a single ribosome along an mRNA molecule as the ribosome translated the mRNA into protein along the length of a hairpin loop (Fig. 6). This type of single-molecule analysis is of particular value because it is impossible to synchronize ribosomal activity, rendering en-

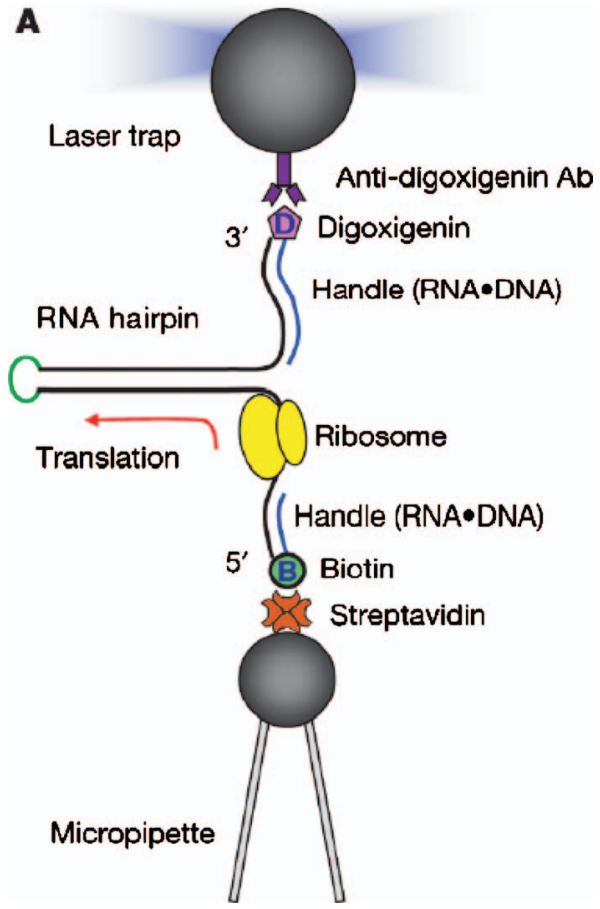

Fig. 6 Configuration used to observe the step size and timing of a single ribosome during translation. An RNA sequence encoding for a hairpin loop was linked to two beads via complementary DNA linker sequences and other chemistries. Ribosome activity was stalled by the omission of aminoacyl-tRNA until the experiment was ready to commence. The RNA sequence was held under a constant tension of $20 \mathrm{pN}$ between a micropipette and an optical tweezers, which was used to measure the forces acted up on the RNA as the hairpin loop unwound due to the ribosomal movement. (Reprinted by permission from Macmillan Publishers Ltd., Nature, Ref. 50, (C) 2008.

semble studies difficult. To achieve this analysis, the authors optically held a single mRNA molecule using bead handles at the terminal ends held under a constant tension force of $20 \mathrm{pN}$. As the ribosome translated along the length of the mRNA molecule through the hairpin loop, it unwound the loop. This manifested itself in the mRNA molecule extending. The extension as a function of time revealed, in detail, the amount of time a ribosome spends translating each codon $(<0.1 \mathrm{~s})$, and additionally demonstrated that each translocation step was equivalent to the distance of three base pairs, or one codon.

\subsection{Protein Folding}

Correct protein folding is of fundamental importance to the viability of a cell, and incorrect folding can results in a variety of disease states. The amino acid sequence of a protein determines its configuration. For example, the hiding of hydrophobic groups minimizes the free energy from the protein-water interaction and is generally the dominant mechanism that drives protein folding. Electrostatic interactions during the folding process, such as salt bridges, hydrogen bonds, or disulfide bonds, limit the number of folding choices as folding ensues. ${ }^{104,105}$ AFM studies have been used to study protein folding, but their much higher spring constants make it difficult to directly observe folding. However, optical tweezers have now been used successfully to study directly this folding 

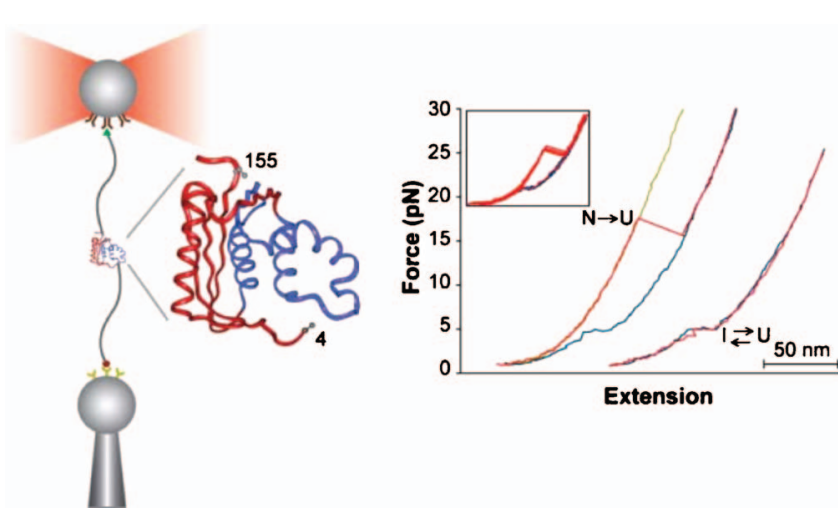

Fig. 7 Single molecule investigation of protein denaturation and refolding. A protein molecule, RNAase $\mathrm{H}$, is attached via cysteine residues to DNA linkers, each of which were attached to two microspheres. Note that the blue region of the protein is the central, stable core region. One of the microspheres was held by capillary suction, and the other by optical tweezers, which were used to generate the force-extension curve on the right. This curve shows the transition of the protein between native $(\mathrm{N})$ to unfolded $(\mathrm{U})$ state during stretching (red line) and relaxation (blue line), as well as a transition between an unfolded (U) and intermediate (I) state at lower forces. Inset: multiple stretching/relaxation curves. The yellow line is a control curve for the DNA linkers. (From Ref. 51. Reprinted with permission from AAAS.)

process. In 2005, Cecconi et al. ${ }^{51}$ analyzed the unfolding and refolding of a small 155 residue protein called RNase $\mathrm{H}$ (Fig. 7). This protein was tethered to two bead handles using two $500 \mathrm{bp}$ dsDNA linkers. Force measurements on one of the beads indicated an intermediate folding state between the native and the unfolded state. As such, this technique can be used to further our understanding of protein-folding dynamics.

\section{Optical Manipulation at the Cellular Level}

Optical traps are not restricted to the world of single molecules. Indeed, they can trap and organize cells, allowing us to understand numerous facets of cell biology. It is worth remarking here that the last decade has witnessed a drive away from single-beam optical tweezers to using multiple optical traps and even more exotic beam shapes, which has had a particular impact on studies with optical traps for cells. Such multiple traps may be created in a variety of ways: timeshared traps can be created with acousto-optic deflectors or galvanometers. Recently, the use of dynamic diffractive optical elements, namely, spatial light modulators, have come to the fore, ${ }^{106,107}$ allowing their use as dynamic holographic elements or to perform more direct imaging via the generalized phase contrast technique. Going beyond a Gaussian light field to other fields, such as Laguerre-Gaussian beams (typically of annular form), or elongated "nondiffracting" Bessel light modes can also offer distinct advantages. For more details on the emergence of advanced beam shaping and multiple traps, there are now a number of specialist reviews available. ${ }^{19,107-109}$

\subsection{Viability of Optically Manipulated Cells}

It is of note that for any optical trapping experiment with cells, ensuring their viability and continued biological func-

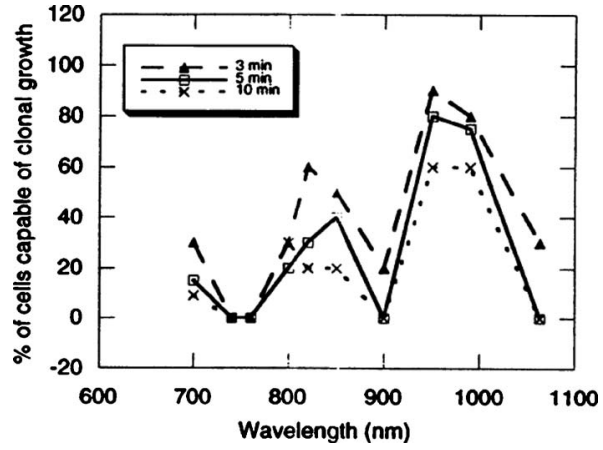

Fig. 8 The viability of $\mathrm{CHO}$ cells in single beam gradient optical tweezers at $176 \mathrm{~mW}$, assessed by colony formation, as a function of the time and wavelength they are held within the beam. Note that these are quite high powers; a lower $88 \mathrm{~mW}$ exposure at $990 \mathrm{~nm}$ for 5 min could result in $100 \%$ viability. (Reprinted from Ref. 110 (C) 1996, with permission from Elsevier.)

tion is a crucial facet to ensure meaningful results. Cell survival during optical manipulation depends on the wavelength, power, the duration of manipulation, the presence of oxygen, and whether the cell has been prestressed.

With regard to eukaryotic cells, one of the most reliable indicators of viability, cloning efficiency, was explored by Liang et al. ${ }^{110}$ to investigate laser related injury (Fig. 8). Using Chinese hamster ovary $(\mathrm{CHO})$ cells, laser-trapping wavelengths ranging from 700 to $1064 \mathrm{~nm}$, and doses ranging from $1 \mathrm{~min}$ at $88 \mathrm{~mW}$ to $3 \mathrm{~min}$ at $176 \mathrm{~mW}$, they found that $990 \mathrm{~nm}$ was the optimum wavelength for tweezing. In contrast, tweezing at $760 \mathrm{~nm}$ resulted in a $100 \%$ loss of viability after as little as $1 \mathrm{~min}$ of exposure to the trapping beam. A separate study using electron microscopy, investigated the response of cells to tweezing for many minutes at a $980 \mathrm{~nm}$ wavelength and low tweezing powers. Primary Ambystoma tigrinum Tiger Salamander neurones showed normal mitochondria, cytoskeleton, cytoplasm, and nucleoplasm. ${ }^{111}$

Prokaryotic cells show a remarkably similar response in viability to different tweezing wavelengths. A study by Neuman et al. explored the viability of Escherichia coli, and found that a wavelength of $970 \mathrm{~nm}$ provided optimum viability. ${ }^{112}$ At this wavelength, cells could be tweezed for $5 \mathrm{~min}$ at $88 \mathrm{~mW}$ without loss of viability. The authors also demonstrated that the loss of viability is exacerbated greatly by the presence of oxygen. Cells tweezed in anaerobic conditions or in the presence of oxygen-scavenging molecules had greatly increased viabilities. They propose that the damage is a one-photon process that may involve singlet oxygen. In another bacterial study using much lower tweezing powers, Rasmussen et al. ${ }^{113}$ used the maintenance of intracellular $\mathrm{pH}$ as an indicator of cell survival during optical tweezing using a $1064 \mathrm{~nm}$ emitting laser and compared four different bacterial species, E. coli, L. monocytogenes, L. inocua, and B. subtilis. At $6 \mathrm{~mW}$, under aerobic conditions, all these species with the exception of $B$. subtilis remained viable for $1 \mathrm{~h}$ or more; under anaerobic conditions, the $B$. inocua strain only survived for $10 \mathrm{~min}$. Three main points were evident from this study: (i) different bacterial species demonstrate different tolerances to optical tweezers, (ii) intraspecies variations also exist, with some subpopulations of cells within a species being more 


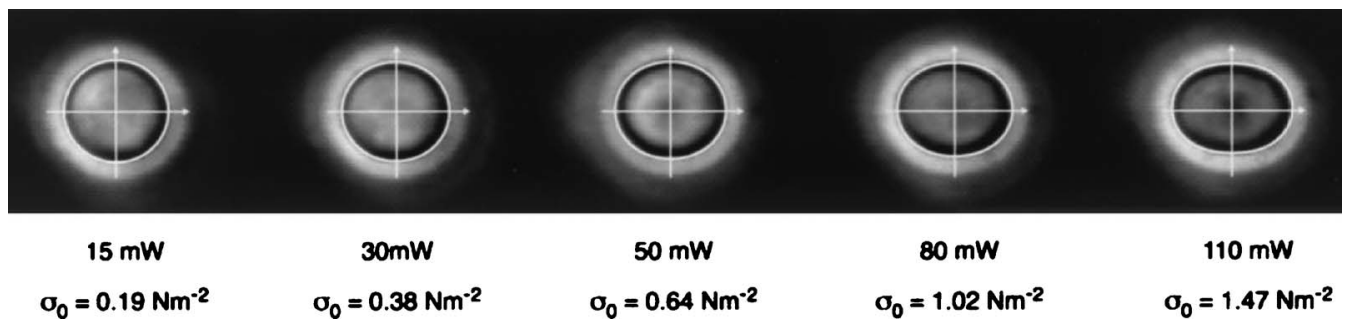

Fig. 9 An erythrocyte being optically stretched in a dual fiber trap. As power increases, the cell becomes more elliptical. Theoretical peak stresses, $\sigma_{\mathrm{o}}$, are also shown. (Reprinted from Ref. 6. ๔ 2001, with permission from Elsevier.)

naturally resistant to tweezing than others, and (iii) aerobic conditions can actually prevent subsequent loss of viability induced by tweezing (especially in L. inocua). Although this latter point seems to be in contradiction to the Neuman study, the experimental conditions were very different (including an eightfold difference in tweezing power and the species of bacteria used to draw this conclusion). It was proposed by the authors that oxidatively stressing cells can potentially confer protection from the subsequent stress of tweezing, ${ }^{113}$ although it could also be the case that hypoxia-induced stress made the cells more sensitive to tweezing.

The idea of prestressing a cell in order to prevent loss of viability during tweezing was also highlighted in a recent study with human breast adenocarcinoma (MCF-7) cells. When cells were preirradiated with a $632.8 \mathrm{~nm}$ source, a significantly greater number of cells survived subsequent optical tweezing at $1064 \mathrm{~nm} .{ }^{114}$ It has been proposed that the mechanism for preirradiation protection may involve activation of the cytochrome c. ${ }^{115}$ Again in mammalian cells, light at optical tweezing wavelengths, has also been shown to induce an expression of heat shock proteins, ${ }^{116}$ which are chaperone molecules that might aid the cell to repair light-denatured proteins.

In spite of these potential limitations, optical tweezers have been used successfully to micromanipulate biological material often in ways that would otherwise be impossible to achieve. Minimizing the time the cell is in the trap, choosing the appropriate wavelength, and using novel tweezers configurations such as the Laguerre-Gaussian (LG) beam ${ }^{117}$ can all serve to minimize photodamage to the biological specimen. A LG mode may offer an annular profile allowing trapping with a lower power than a Gaussian beam counterpart and exposing less of the intracellular volume of a cell to high fluences. Overall, these studies highlight the importance of choice of laser wavelength and beam shape in trapping studies of cells. As a summary, although the "therapeutic window" of tweezing wavelengths has classically been considered to be between 700 and $1064 \mathrm{~nm}$, the viability of tweezed cells changes dramatically with wavelength. For mammalian cells tweezed within this wavelength range, maximum viability lies between 950 and $990 \mathrm{~nm}$ (with 800-850 nm also demonstrating a smaller maximum) and minimum viability is between 740 and $760 \mathrm{~nm}^{110}$ For prokaryotic cells tweezed within the range $790-1064 \mathrm{~nm}$, maximum viability is observed at $970 \mathrm{~nm}$ (with a smaller maximum at $830 \mathrm{~nm}$ ) and minima are observed at 870 and $930 \mathrm{~nm} .{ }^{12}$ Tweezing with the popular $1064 \mathrm{~nm} \mathrm{Nd:YAG} \mathrm{source} \mathrm{is} \mathrm{not} \mathrm{necessarily} \mathrm{the}$ most appropriate wavelength choice if subsequent subculturing is desired.

\subsection{Optical Stretching}

Emergent studies at the single-cell level have included the development of optical stretching of cells. The dual counterpropagating trap arrangement leads, in fact, to a bulging of a trapped deformable object of higher refractive index than its surroundings. This is a consequence of the change in photon momentum during passage from a medium of lower to higher refractive index - that is, as the light passes from the medium into the trapped object and reemerges on the other side. The direction of the force at the cell-medium interface can be readily derived using the Minkowski formulation. ${ }^{6}$ An indepth discussion of the deformation of soft materials by optical tweezers was presented by Møller and Oddershede. ${ }^{118} \mathrm{In}$ the case of a cell, the bulging and elliptical shape can be used to infer the state of the cytoskeleton. This is an internal polymer structure that can resist external optical forces. The optical stretcher allows the recording of the cell's ellipticity and thus informs on when it changes (Fig. 9). ${ }^{6}$ The original cellstretching work looked at the elasticity of erythrocyte (red blood cell) and fibroblast BALB 3 T3 cells. ${ }^{6}$ Subsequent work has employed optical stretching as a biological marker for cancer. Essentially, neoplastic and diseased cells have weaker cytoskeletons and thus show on average larger deformations/ ellipticity than normal cells. Thus, recording the distribution of a cell's ellipticity can be indicative of the onset of disease. In their 2005 study, Guck et al. compared the elasticity of a variety of healthy and cancerous cells lines, including models of breast cancer, and found that the cancerous cells were significantly more deformable than their noncancerous counterparts. ${ }^{119}$ Furthermore, cancer cells treated to either increase or decrease their metastatic competence had a corresponding increase or decrease in deformability. Subsequent work revealed a significant increase in the deformability of acute promyelocytic leukemia ${ }^{120}$ and oral squamous cell carcinoma $^{121}$ cell types when compared to their noncancerous counterparts, further confirming the diagnostic potential of the technique.

As well as the dual counterpropagating trap, optical stretching can also be performed with single-beam optical tweezers. Erythrocytes and yeast cells are among common cell types studied with optical tweezers. ${ }^{22,76,107}$ Liao et al. ${ }^{122}$ describe an optical stretcher based on an acousto-optic modulator, which was used to scan the focal point of the tweezers between two ends of an erythrocyte at a rate of $100 \mathrm{~Hz}$. As a 

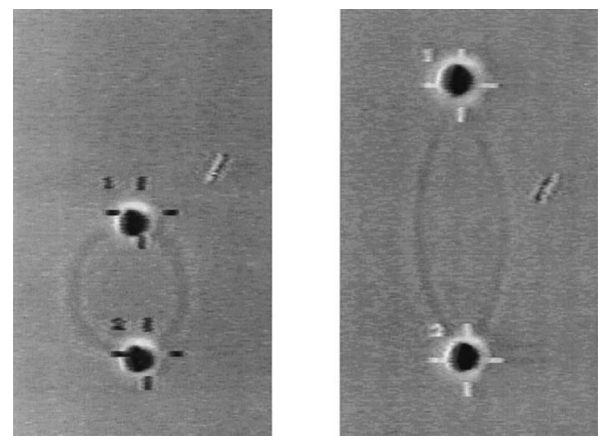

Fig. 10 A red cell ghost being before and during optically stretching. The cell was treated with $\mathrm{N}$-ethylmaleimide prior to stretching, which dissociated spectrin tetramers to dimers. (Reprinted from Ref. 125. (c) 1999, with permission from Elsevier.)

result of the viscous drag imparted by the solution on the cell, instead of hopping between the two traps, the cell remains stationary and becomes stretched up to $0.5 \mu \mathrm{m}$ in extra length. ${ }^{122}$ Erythrocytes have also been optically stretched using microspheres acting as "handles" on opposite ends of the cell. This was first described by Bronkorst et al., ${ }^{123}$ and shortly thereafter used by Sleep et al. ${ }^{124}$ to study the elasticity of erythrocyte membranes after chemical disruption of spectrin, ankyrin, or actin (Fig. 10). More recently, Suresh et al. ${ }^{125}$ used optical tweezers to measure the elastic modulus of erythrocytes during various stages of infection by the malarial parasite $P$. falciparum. As an infection progresses, cells become less deformable and more adhesive. The advantage of this optical technique is that during the final (schizont) stage of infection, this morphological change makes it difficult to measure the elasticity of cells using classical micropipette aspiration or laminar shear flow methods. ${ }^{125}$

Whole cell mechanics aside, the mechanical properties of small intracellular regions can also be analyzed. Wei et al. performed a study of viscoelasticity of alveolar epithelial cells using a piezoelectric-driven oscillating optical tweezers and compared two methods: tweezing extracellularly bound (via an antigen-antibody interaction) $1.5 \mu \mathrm{m}$ spheres, and tweezing endogenous intracellular granules. ${ }^{126}$ In this work, the amplitude of the particle displacement and its phase shift, both of which are frequency dependent, were used to deduce the spring constant of the trap, and from there the magnitude of the complex modulus $\left|\mathrm{G}^{*}\right|$ was calculated. The frequencydependent complex modulus $\mathrm{G}^{*}$ is a combination of the materials elastic $\left[\mathrm{G}^{\prime}(\omega)\right.$-the shear storage modulus] and viscous $\left[G^{\prime \prime}(\omega)\right.$-the loss storage modulus] response, with $\mathrm{G}^{*}(\omega)=\mathrm{G}^{\prime}(\omega)+i \mathrm{G}^{\prime \prime}(\omega)$. The authors demonstrated that significantly lower internal storage and shear moduli were measured using the intracellular granules than the extracellular beads.

\subsection{Trapping for Cell Biology and Microfluidic Environments}

Gene technology has advanced to such an extent that we can alter a single cell's function. The ability to record the response of single cells to a range of gene manipulation opens up a new avenue for establishing more knowledge about individual cel- lular functions. Microfluidic systems, typically a series of channels with diameters of up to $100 \mu \mathrm{m}$, can assist in such studies and can mimic in vivo conditions by allowing cells to be exposed to different biochemical media. The physics of such narrow channels allows laminar flow (low Reynolds number regime) with little mixing. Optical forces can play a pivotal role in such geometries because they can controllably transport a cell between different chemical environments. This ability has been demonstrated by a number of groups, and this type of in situ assay allows the real-time analysis of fluorescent, Raman, or morphological changes in response to a changing chemical environment. For example, as described below, cells can be tweezed from different chambers or between laminar flow streams in order to dose them with different reagents, all during microscopic examination. Alternatively, microdroplets containing femtoliter-attoliter volumes can be tweezed to deliver reagents to a specified cell, or to extract, transport, or analyze subcellular vesicles or lysates.

In the study by Eriksson et al., flow velocities had to be high enough that the reagents did not appreciably mix, but low enough that the cells were not displaced from the trap by shear. ${ }^{127}$ The studies revealed real-time changes in gene expression in response to the tweezing between different reagent streams. In one example, the nuclear localization of Yap1p, a transcription factor involved with the induction of defense against oxidative stress, was observed in response to the tweezing of a BY4741 wild-type yeast cell from normal cell culture medium to the oxidatively stressing tert-butyl hydroperoxide containing medium.

Other cellular experiments have been demonstrated without microfluidics but still exposing the cells to different media. Capitalizing on the slow diffusion rate of solutes out of $4 \mathrm{~mm}$ long, $50 \mu \mathrm{m}$ id. capillary tubes, Brown et al. demonstrated the optical tweezing of single HL60 cells between three different reagents: fixative, permeabilization buffer, and Trypan Blue. ${ }^{128}$ Indeed, recent work has used optical forces in a new way, employing Airy light fields and moving on parabolic trajectories to transfer cells from one medium to another in the absence of any microfluidic flow. ${ }^{129}$

Liposomes and other micro- and nanoparticles commonly used for the transfection of cells are also tweezable vehicles for targeted reagent delivery. Once tweezed in close proximity, contents can be either be released or injected into the cell. ${ }^{130}$ In one chemical release experiment, optical tweezed particles containing carbachol (a ligand for the muscarinic acetylcholine receptor) were lysed in close proximity of a cell expressing the receptor, using a pulsed UV laser (Fig. 11). Activation of the receptor was assessed fluorescently. ${ }^{131,132}$ The controlled release of a compound in this manner is highly desirable as localized concentration gradients of diffusible chemotactic agents that are ubiquitous control signals for cell migration, differentiation, developmental biology, and the immune system. This type of experiment is traditionally performed using a finely extruded glass capillary tube, ${ }^{133}$ but the advantage with using optical tweezers is that it would provide far greater spatial and temporal control of the released reagent. Another excellent example was recently demonstrated by Kress et al., who used arrays of holographic optical tweezers to release compounds from optically manipulated microparticles. ${ }^{134}$ By releasing chemoattractants, they demonstrated the polarization and directed migration of a neutrophil 


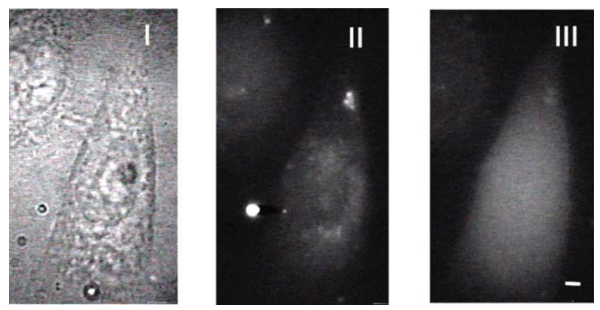

Fig. 11 A polystyrene particle containing carbachol (white dot, II) was optically tweezed to a cell that was expressing the muscarinic acetylcholine receptor (of which carbachol is a ligand) and that was preloaded with fluo-3 (which fluoresces proportionally to intracellular calcium release). Upon photolysis of the carbachol containing particle by UV pulse, the highly localized drug caused an increase in intracellular calcium (III). (Reprinted with permission from Ref. 133. (c) 2004 American Chemical Society.)

and the generation of new lamellipodia in HL60 cells. By releasing inhibitors of actin polymerization, they demonstrated the highly localized retraction of lamellipodia and the redirection of migration in the same cell type. In effect, this robust technique allows the full optical control of cell migration.

Finally, the activation dynamics of membrane receptors using microbeads that are coated with ligands can be studied using optical tweezers. Monneret et al. used holographic optical traps to maneuver various microparticles to specific regions of individually targeted COS-7 cells suspended within a microfluidic environment. ${ }^{135}$

\subsection{Imaging and Spectroscopy with Optical Manipulation}

A theme that has emerged in the last decade has been the combination of an optical trapping with other optical methods, namely, imaging and spectroscopic analysis. This ability is essential if we wish to monitor and observe the cellular behavior over long time courses, and indeed, such combinations of techniques are emerging as forerunners for redefining modern microscopes: a future "biophotonics workstation" may be capable of a plurality of studies on one platform.

In terms of imaging, optical tweezers have been combined with imaging by epi-fluorescence ${ }^{127}$ confocal laser-scanning microscopy (CLSM), ${ }^{136}$ and multiphoton microscopy. ${ }^{137}$ Fluorescent systems can either be homebuilt, or commercially available fluorescent microscopy platforms can be modified to incorporate tweezing beams. ${ }^{44}$ The Hoffmann study was one of the first of its kind to address the technical challenge of implementing CLSM and optical tweezers via the same microscope objective. ${ }^{136}$ During CLSM, the microscope stage is moved axially (i.e., in $Z$ ) in order to obtain scanned $X Y$ 2-D image slices, which are later reconstructed into a 3-D image. The image plane remains a fixed axial distance from the objective. When a sample object is being optically tweezed, however, it also remains at a fixed distance relative to the objective. The two relative axial positions of the CLSM and the tweezers therefore had to be decoupled. To achieve this, the authors placed a translation stage onto the source of the trapping beam, changing the relative distance between it and the first lens in the system. This resulted in the ability to axially shift the optical tweezers focal plane relative to the
CLSM focal plane by $15 \mu \mathrm{m}( \pm 0.25 \mu \mathrm{m})$. The simultaneous tweezing and confocal imaging of chloroplasts and cancer cells was demonstrated. Using a slightly different configuration, Goksör et al. demonstrated the simultaneous multiphoton $^{137}$ imaging and tweezing of yeast cells. The group further demonstrated the versatility of its multiphoton imaging and trapping configuration by holding a single spermatozoon with a continuous wave 1064 beam, optically injecting a membrane impermeable dye into the spermatozoon cell using a femtosecond-pulsed $800 \mathrm{~nm}$ source, and imaging the entry of the dye over time using epi-fluorescence.

In what other contexts can this combined fluorescent imaging and tweezing be useful? Oddos et al. demonstrated the imaging of a synapse formed between an immune cell and an antigen presenting cell. ${ }^{138}$ This synapse was difficult to image with high resolution under normal microscopy conditions because the two cells naturally resided in the horizontal position. The authors capitalized on the fact that a cell couplet when optically tweezed assumed a vertical position. This vertical positioning allowed the rapid 2-D imaging of the immune synapse at the exact plane of the two contacting cells. Without the use of tweezers to position the couplet vertically, CLSM would otherwise require multiple $\mathrm{Z}$ slices to reconstruct the synapse, not only reducing the image resolution but also significantly increasing the acquisition time.

In another interesting biological application, Hayakawa et al. ${ }^{139}$ used standard optical tweezers and CLSM to investigate the activation of mechano-sensitive channels. In an elegant experiment, they microinjected $400 \mathrm{~nm}$ phalloidin-conjugated beads into live HUVEC cells. The beads selectively bound to the actin cytoskeleton. By tweezing the beads with single beam gradient tweezers, they were able to demonstrate by patch clamping, that the mechanical force acting upon the actin results in mechano-sensitive channel activation. In contrast, the tweezing of microinjected control beads that were not tagged with phalloidin did not result mechanosensitive channel activation (Fig. 12). ${ }^{139}$

Combining fluorescent imaging with holographic optical tweezing has also been demonstrated. Eriksson et al. showed the ability to manipulate dozens of individual yeast cells such that the nuclei of each cell was in the correctly focussed imaging plane. ${ }^{140}$ In other words, instead of imaging 3-D stacks of a large number of cells resting on a glass coverslip, the cells were manipulated above the coverslip into free solution and automatically repositioned axially such that all nuclei could be imaged concurrently. In the future, this type of configuration could allow the real-time in situ analysis of dozens of trapped cells in response to a drug challenge, for example.

In terms of spectroscopy, Raman spectroscopy has been successfully combined with optical traps for a variety of studies. Raman scattering is an inelastic process and generates a biochemical signature of the molecules. However, the Raman signal is very weak (for example, compared to fluorescence) and can be hampered by the immediate environment of the cell. In this regard, combining this method with trapping thus assists in isolating the cell from any local surface (e.g., a coverslip). Additionally, the cell can be exposed to different buffer media and the Raman signal recorded in situ, or the cell can be rotated and oriented as a Raman signal is recorded from different subcellular regions. Most molecules are termed Raman active and the process requires no external labelling or 


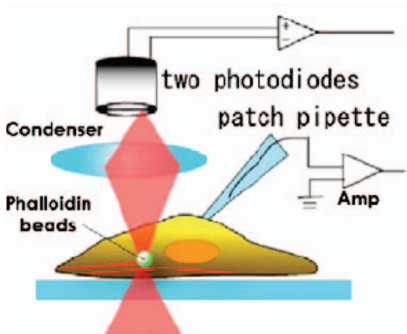

(a)

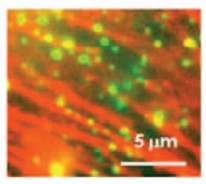

(b)

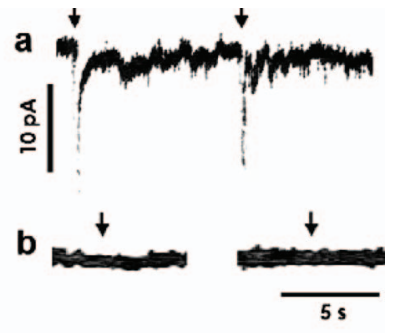

(c)

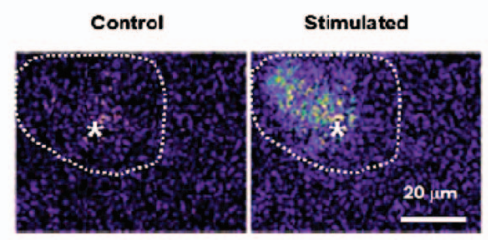

(d)
Fig. 12 Mechanosensitive channel activation by the mechanical stimulation of actin fibres via optical tweezers. (a) Phalloidin laden $40 \mathrm{~nm}$ green fluorescent beads were microinjected into HUVEC cells, (b) which bound to actin stress fibers (red) observable under CLSM. (c) a: a transient inward current occurred (arrows) when beads were moved using optical tweezers, b: but no current was present in moved control beads that were not actin bound. (d) This mechanical stimulation via optical tweezers also caused an increase in intracellular calcium $(*)$ in the HUVEC cell (outlined with dotted lines) visualized by CLSM. [Reproduced with permission from the Journal of Cell Science http://dx.doi.org/10.1242/jcs.022053 (Ref. 140)].

fluorophores making it attractive. Raman studies with optical trapping ${ }^{141}$ originated in 1994 and have found major applications for biomedical problems. In addition, innovation in the Raman trapping apparatus has also been pursued. Geometries have been explored both using the same beam for trapping and Raman, and decoupling these beams. Additionally, both holographically generated multipoint traps and counterpropagating dual-beam traps have been used. This latter trap was used to immobilize large cells for Raman analysis. Jess et al. ${ }^{8}$ used this system and showed Raman signatures from the cytoplasm, nucleus, and membrane, and the ability to discriminate between them.

In 2002, a single-beam gradient optical tweezers holding individual cells in place while simultaneously taking Raman spectra was demonstrated. The system operated at $785 \mathrm{~nm}$ using the same laser for trapping and Raman analysis. It was calibrated by use of polystyrene microbeads and tested on living blood cells and on both living and dead yeast cells. As expected, different Raman spectra were observed for the different cells. ${ }^{142}$ The same team extended its work to look at sorting, using Raman identification. ${ }^{143}$ In separate work by Creely and colleagues, ${ }^{144}$ T-lymphocytes in suspension were held in place using holographic optical tweezers while being probed with a second Raman beam.

Raman may also be extended to look at cell disorders, disease, and even the onset of cancer. Thus early work by Zheng et al. ${ }^{145}$ describe the potential of Raman tweezers in cancer diagnosis. They used Raman tweezers to analyze 200 normal and 200 cancerous colorectal cells and perform multivariate analysis for discrimination. Promising results for specificity and sensitivity were obtained in this work. Raman spectra from optically trapped cancerous and normal colorectal epithelial cells were also obtained by Chen and colleagues who aimed to investigate intact cells. ${ }^{146}$ This study had clinical relevance because patients suffering from colorectal adenocarcinomas supplied the cell suspensions for this work. The Raman spectra indicated higher amounts of nucleic acids and proteins in the cancerous cells, which is consistent with other studies. Recent studies by Harvey et al. looked at Raman tweezers as a tool to explore urological cell lines, with the aim of distinguishing malignant cells with principal component analysis. ${ }^{147}$

Biological studies of erythrocytes and lymphocytes have been a prominent topic combining Raman and trapping. Such cells have major biological relevance for diseases such as leukemia ${ }^{148}$ as well as being typically smaller than epithelial cells, thus making them more amenable to trapping. Another advantage using hematopoietic cells in this type of experimentation is that they do not readily adhere to surfaces, simplifying their preparation for tweezing. Finally, the porphyrin ring contained in hemoglobin strongly absorbs visible light, enhancing resonance and allowing the oxygenation and spin state of erythrocytes to be easily monitored. ${ }^{149}$ Ramser et al. explored the oxygenation state of an erythrocyte. ${ }^{150}$ Using a microfluidics platform, different buffers passed through the channels using electro-osmotic flow. At the same time, the resonance Raman response of an optically trapped erythrocyte was recorded. This enabled them to monitor the oxygenation cycle of the cell in real time and to study the photoinduced chemistry caused by the chamber illumination, but naturally this could be extended to other biological end points. Chan et al. showed highly reproducible Raman spectra could be obtained by trapping live cells obtained from leukemia patients; multivariate analysis could be used for distinguishing normal and abnormal cells. ${ }^{151}$ Other studies explored the oxygenation capability of diseased erythrocytes: Rusciano et al. explored the characterization of an erythrocyte from $\beta$-thalassemic patients obtained by using an optical tweezers system integrated with a Raman probe. ${ }^{152}$ They compared the Raman spectra of single thalassemic erythrocytes to those obtained from healthy subjects, relating the observed differences to the analyzed disease. The photoinduced effects caused by the Raman and trapping laser radiation were also investigated. The oxygenation capability of hemoglobin was shown to be reduced in $\beta$-thalassemic erythrocytes. In other studies, Raman spectroscopy monitored changes in the oxygenation state of human erythrocytes placed under mechanical stress by optical forces leading to cell stretching. The optical force in this instance mimicked the stretching and compression that flowing cells undergo as they flow along smaller capillaries. Rao et al. ${ }^{153}$ showed spectroscopic evidence of a transition between the oxygenation and deoxygenation states, which is induced by stretching the cell with optical tweezers. They attributed the transition to enhanced hemoglobin-membrane and hemoglobin neighbor-neighbor interactions. ${ }^{153}$ This has just given a flavor of the area of Raman spectroscopy and optical trapping. For the interested reader, there are more in-depth reviews of this field. ${ }^{154-156}$

\subsection{Tweezers and Nanosurgery}

The application of manipulation to cellular material is achieved with minimal damage from the trapping beam. How- 
ever, it can be advantageous to combine optical tweezers with other light fields that are designed to perform very precise cutting or controlled transient perforation with submicron resolution. Berns explained the use and history of optical scissors in Ref. 157.

UV laser fields, pulsed Nd:YAG lasers, or ultrashort pulsed fs beams may typically be employed to function as "optical scissors." Such scissors were employed to study the intracellular organelles, including chromosomes, and the mitotic spindle. In 1991, Steubing et al. ${ }^{158}$ brought two cells into contact using an optical trap. The application of a UV laser microbeam then cut the common wall of the two cells allowing them to fuse. ${ }^{158}$ This enabled the creation of hybrid cells that retained cell function. In other work, Seeger et al. ${ }^{159}$ used a laser microbeam to microdissect chromosomes collected by an optical trap and subsequently collected the chromosome segments using a glass capillary. ${ }^{159}$ Similar methods can be used for the dissection of cellular organelles, cytoskeletal filaments, ${ }^{160}$ or chromosomes. ${ }^{161-164}$ More recently, such systems can be used for the separation of individual cells from cell clusters. ${ }^{165}$ Finally, the cell membrane may also be transiently permeabilized by applying CW or pulsed light, leading to cell transfection. ${ }^{166}$

Large liposomes (up to $50 \mu \mathrm{m}$ diam) may be used to perform chemical reactions in closed nanovials using only picoliters of reagent. Kulin et al. ${ }^{167}$ used optical tweezers to trap two individual liposomes, which were drawn then into contact. A "nanosurgery" beam, in this case a single pulse of ultraviolet laser light induces the fusion between liposomes. ${ }^{167}$ This allowed reagents held within the liposomes to mix and react with one another. Such studies may enable quantitative studies of mixing of chemicals and combinatorial chemistry with picolitres of reagents.

Such nanosurgery may also facilitate the analysis of subcellular components. For example, vesicles can be extracted from cells and optically tweezed to a destination for proteomic $^{168-170}$ or genomic ${ }^{165}$ analysis. As a parallel example, lipid-coated oil droplets can be tweezed to the plasma membrane of a cell and used to extract membrane components. ${ }^{171}$ Once extracted, subcellular material can be further processed using tweezable microdroplet reactors. ${ }^{167,172,173}$ It is key to note that the handling of single subcellular components, such as mitochondria, endoplasmic reticulum, endosomes, etc., is technically challenging using other methods such as by an extruded capillary tube. Therefore, optical tweezers will no doubt continue to play a valuable role in the transport and analysis of these precious samples.

\subsection{Cell Patterning and Directed Growth}

Since Ashkin and Dziedzic first demonstrated the tweezing of a virion and a bacterium, ${ }^{174}$ optical manipulation has found a variety of applications in tissue engineering and neuroscience where, in particular, patterning and organization of biological material is essential. 2-D and 3-D patterns of nerves have been demonstrated, ${ }^{175}$ sometimes with the goal of generating artificial neural networks. ${ }^{111}$ In 1998, Townes-Anderson et al. tweezed a rod cell, cone cell, and multipolar neuron (cells all from the retina) adjacent to each other. Subsequently, new processes interconnecting these cells were subsequently

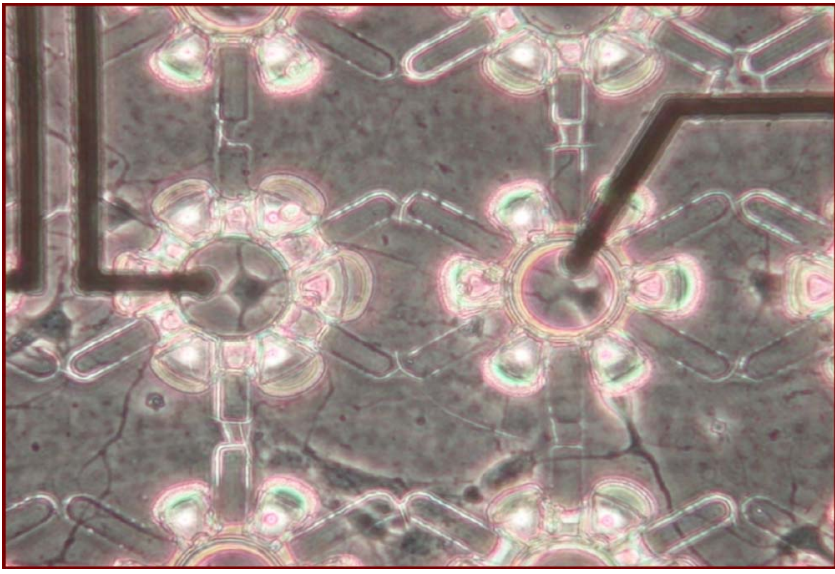

Fig. 13 Parlene neurocages, designed to hold, electrically stimulate, and record the activity of individual neurones, ${ }^{177}$ are seeded one cell at a time using optical tweezers. [Image reproduced from Ref. 178 with permission (C 2009 IEEE)].

formed. ${ }^{111}$ Manipulation by optical tweezers can also be used to deliver single cells to microfabricated sensors ${ }^{176,177}$ or microwells. ${ }^{178}$ In 2009, Pine and Chow demonstrated the delivery of rat hippocampal neurones to parlene plastic cages containing electrodes for stimulation and recording, ${ }^{177}$ opening up a new type of electrophysiological experimentation (Fig. 13). Other studies have investigated heterotypic cell-cell patterning on a larger scale for tissue engineering. In 2006, Akselrod et al. ${ }^{179}$ used a combination of a SLM and an AOD to generate arrays of live cells onto a photopolymerizable polyethylene glycol diacrylate hydrogel. 2 -D $(21 \times 21$ matrix $)$ or 3 -D $(3 \times 3 \times 3$ matrix $)$ arrays of $P$. aeruginosa bacteria were produced, which could be subsequently fixed into position using the photopolymerizable gel. Heterotypic arrays of Swiss 3T3 cells and bacteria were also generated.

The real-time optical control of cell growth has also been demonstrated. Both the axons of a growing neuron and the hyphal tip of fungi have been optically controlled using optical tweezers. Burnham et al. ${ }^{180}$ demonstrated the controlled growth of the fungal hyphae using engineered light fields to constrict and induce branching in a highly reproducible manner. ${ }^{180}$ Light fields have also been used to guide the axons of mammalian PC-12 and NG-108 cells. ${ }^{181-184}$ These experiments are surprising, and the underlying mechanisms are not fully understood.

This precise cellular patterning and cell delivery using lasers may have future consequences in tissue engineering. It is well established that the microenvironment of a cell greatly influences its state of differentiation, and one of the great challenges of tissue engineering has been the precise control of cell-cell contacts. ${ }^{185}$ One classic example is the primary hepatocyte, which rapidly loses a number of hepatocytespecific markers, such as cytochrome P- $450^{186}$, albumin, and urea $^{187}$ once removed from its natural environment. This loss of function can be ameliorated by coculture with nonparenchymal cells. ${ }^{188}$ Using a weakly focussed $830 \mathrm{~nm}$ wavelength beam, Nahmias et al. explored tissue engineering possibilities using laser cell patterning. ${ }^{189}$ By carefully depositing either one cell type (homotypic) or two different cell types (hetero- 
typic) in linear arrays, cells spontaneously aggregated into tissuelike structures. In the former instance, laser-deposited human umbilical vein endothelial cells (HUVEC) selfassembled into vascular (blood-vessel-like) structures. In the latter case, a combination of primary rat hepatocytes heterotypically laser patterned with HUVEC cells resulted in tubular structures reminiscent of the hepatic sinusoid, a highly specialized liver blood vessel. Therefore, the ability to pattern cocultured cells using optical tweezers may help elucidate the mechanisms of phenotype maintenance in all cell types such as hepatocytes, neurons, and potentially stem cells.

Arneborg et al. used cell patterning to observe synergistic yeast ecosystem dynamics. ${ }^{190}$ The authors organized two species of yeast cells to study their growth characteristics using the generalized phase contrast technique as their multiple-trap generation method. In this study, Hanseniaspora uvarum cells were surrounded by Saccharomyces cerevisiae cells. The $S$. cerevisiae cells indeed influenced the growth of H. uvarum. The generation time of surrounded $H$. uvarum cells was $15 \%$ greater than that of nonsurrounded cells. The confinement imposed by viable $S$. cerevisiae cells on $H$. uvarum led to a significant proliferation of the latter cell. The conclusion was that such confinement determined growth in a microbial ecosystem.

\subsection{Cell Sorting}

Since its invention in $1965,{ }^{191}$ fluorescence-activated cell sorting has been a powerful technique in the separation of fluorescently tagged cells. In this technique, cells are hydrodynamically focused so they pass through an orthogonally directed laser beam surrounded by detectors. A combination of forward scatter, side scatter, and fluorescence is measured. The flow stream is then split into droplets (with approximately one cell per droplet), and an electrostatic charge proportional to the measured fluorescence is placed on the cell containing droplet. The charged droplets may then be electrostatically separated.

There is a continuing trend to miniaturize this device to an on-chip system. The sorting of cell types, particularly in a microfluidics environment, is another major application of optical forces at the single-cell scale. Here tweezers can "actively" drag biologically tagged cells from a flow stream, creating a microscopic fluorescence activated cell sorter ${ }^{192}$ or in fact aim to sort cells on a potential energy landscape by relying on differing physical attributes (e.g., size) of one cell type compared to another. ${ }^{193}$ One example can be found in the work of Perroud et al. who used a microfluidic system in combination with optical tweezers in order to separate fluorescently green labeled macrophages from a mixed cell population. ${ }^{194}$ High purities (93\%) were reported with reasonable recoveries (39-60\%). More recent studies have explored techniques of passive optical sorting where the very shape or size difference between cells can be used to sort cells. Arrays of optical traps create what are termed optical potential energy landscapes. ${ }^{195,196}$ Optical potential energy landscapes are in a key position to aid with the trend in miniaturization particularly in the field of optical sorting and separation. Motion of biological material across such a landscape depends crucially on the interplay between the physical attributes of the particle and the forces exerted by the landscape, which depend not only on laser power but on its optical form (e.g., periodicity). One example of an optical landscape that can be used for cell sorting is the Bessel beam. A Bessel beam offers the profile of a central "nondiffracting" maximum of light surrounded by a series of concentric rings. It is typically produced by sending a Gaussian beam through a diffractive optical element termed a conical axicon lens or may be generated with a spatial light modulator. Paterson et al. ${ }^{196}$ demonstrated the passive optical sorting of erythrocytes and lymphocytes. In the Bessel beam, the erythrocytes become trapped in the concentric rings, whereas the lymphocytes migrate to the central core of the beam and are subsequently guided along its axis. ${ }^{196}$ Mononuclear and sphere-tagged T-lymphocytes have also been sorted in this manner. ${ }^{197}$

A further separation technology of note is that of optical chromatography, which separates particles or cells based on a competing drag force from a fluid flow and radiation pressure from a laser. ${ }^{198}$ As particles flow in solution toward a weakly focused beam, they are trapped by the beam. Depending on their size and refractive index, they will be trapped in different positions along the length of the light field, allowing extremely sensitive separation to occur. In this technique, stokes drag is balanced by radiation pressure; particles with a higher refractive index and a larger size will be affected more strongly by the radiation pressure than the stokes drag. This configuration has recently realized the separation of $B$. anthracis from a genetically related spore, B. thuringiensis. ${ }^{199} \mathrm{~A}$ mixed spore population of the two species were flowed along a microfluidic channel against the optical pressure of a loosely focussed $850 \mathrm{~nm}$ laser. The $B$. anthracis strain was affected more strongly by the radiation pressure of the laser and was therefore separated many hundreds of microns upstream from the $B$. thuringiensis spore. Optical chromatography has also been used for highly sensitive immunodetection. Antibodycoated spheres, when challenged with antigen, bound to each other as couplets. These couplets were then able to be separated by optical chromatography from unbound spheres; ${ }^{200}$ the ratio of the separated singlet and couplet spheres is proportional to the amount of antigen present. ${ }^{201}$

\section{Conclusions}

Forty years have now passed since Ashkin's first experimental demonstration of an optical trap. ${ }^{4}$ Few could have predicted the immense impact that optical forces have had in biophotonics. In particular, the ultrasmall controllable and measurable forces light can exert has enabled a number of landmark studies for macromolecules and cells and this will undoubtedly continue. Microscopy platforms of the future combining advanced photonic control of the microenvironment of mammalian cells and their single-molecule components will allow us to understand, diagnose, treat, and reverse engineer fundamental biological processes and disease. Advances in singlemolecule techniques and instrumentation will allow an increasing understanding of the invisible nanoscopic world. In cell biology, common laboratory protocols that require millions of cells for an answer are being scaled down to the level of the single cell. Optical forces and traps will play a central role is such studies. As we move forward and embrace new advances, such as precision tracking of particles using fast camera technology, dynamic diffractive optics, and the tech- 
niques of soft lithography for generating ultraprecise channels, we will witness a sustained and enhanced rate of new studies enabled by optical forces. Light will certainly force the pace in years to come.

\section{Acknowledgments}

We thank the UK Engineering and Physical Sciences Research Council for Funding and the EU Network of Excellence PHOTONICS4LIFE. K.D. is a Royal Society Wolfson Merit Award Holder.

\section{References}

1. J. Kepler, De cometis libelli tres, typis Andreae Apergeri, sumptibus Sebastiani Mylii bibliopolae augustani, Avgvstae Vindelicorum (1619).

2. P. Lebedew, "Untersuchungen über die Druckkräfte des Lichtes," Ann. Phys. 311(11), 433-458 (1901).

3. E. F. Nichols and G. F. Hull, "The pressure due to radiation," Astrophys. J. 17(5), 315-351 (1903).

4. A. Ashkin, "Acceleration and trapping of particles by radiation pressure," Phys. Rev. Lett. 24(4), 156-159 (1970).

5. A. Constable, J. Kim, J. Mervis, F. Zarinetchi, and M. Prentiss, "Demonstration of a fiber-optical light-force trap," Opt. Lett. 18(21), 1867-1869 (1993).

6. J. Guck, R. Ananthakrishnan, H. Mahmood, T. J. Moon, C. C. Cunningham, and J. Käs, "The optical stretcher: a novel laser tool to micromanipulate cells," Biophys. J. 81(2), 767-784 (2001).

7. P. B. Bareil, Y. Sheng, and A. Chiou, "Local scattering stress distribution on surface of a spherical cell in optical stretcher," Opt. Express 14(25), 12503-12509 (2006).

8. P. R. T. Jess, V. Garcés-Chávez, D. Smith, M. Mazilu, L. Paterson, A. Riches, C. S. Herrington, W. Sibbett, and K. Dholakia, "Dual beam fibre trap for Raman microspectroscopy of single cells," Opt. Express 14(12), 5779-5791 (2006).

9. W. Singer, M. Frick, S. Bernet, and M. Ritsch-Marte, "Self-organized array of regularly spaced microbeads in a fiber-optical trap," J. Opt. Soc. Am. 20(7), 1568-1574 (2003).

10. A. Ashkin and J. M. Dziedzic, "Stability of optical levitation by radiation pressure," Appl. Phys. Lett. 24(12), 586-588 (1974).

11. K. T. Gahagan and G. A. Swartzlander, Jr., "Optical vortex trapping of particles," Opt. Lett. 21(11), 827-829 (1996).

12. K. Sasaki, M. Koshioka, H. Misawa, N. Kitamura, and H. Masuhara, "Optical trapping of a metal-particle and a water droplet by a scanning laser-beam," Appl. Phys. Lett. 60(7), 807-809 (1992).

13. A. Ashkin, J.-M. Dziedzic, J. E. Bjorkholm, and S. Chu, "Observation of a single-beam gradient force optical trap for dielectric particles," Opt. Lett. 11(5), 288-290 (1986).

14. W. D. Phillips, "Nobel Lecture: laser cooling and trapping of neutral atoms," Rev. Mod Phys. 70(3), 721-741 (1998).

15. H. J. Metcalf and P. van der Straten, Laser Cooling and Trapping, Springer, New York (1999).

16. R. Grimm and M. Weidemuller, "Optical dipole traps for neutral atoms," in Advances in Atomic, Molecular, and Optical Physics, B. Bederson and H. Walther, Eds., pp. 95-170, Academic Press, New York (1999).

17. K. Dholakia, P. Reece, and M. Gu, "Optical micromanipulation," Chem. Soc. Rev. 37, 42-55 (2008).

18. D. G. Grier, "A revolution in optical manipulation," Nature 424(6950), 810-816 (2003).

19. A. Jonáš and P. Zemánek, "Light at work: the use of optical forces for particle manipulation, sorting, and analysis," Electrophoresis 29(24), 4813-4851 (2008).

20. K. C. Neuman and S. M. Block, “Optical trapping," Rev. Sci. Instrum. 75(9), 2787-2809 (2004).

21. K. Ramser and D. Hanstorp, "Optical manipulation for single-cell studies," J Biophoton. 3(4), 187-206 (2009).

22. H. Zhang and K. K. Liu, "Optical tweezers for single cells," J. R. Soc., Interface 5(24), 671-690 (2008).

23. W. M. Greene, R. E. Spjut, E. Bar-Ziv, A. F. Sarofim, and J. P. Longwell, "Photophoresis of irradiated spheres: absorption centers," J. Opt. Soc. Am. B 2(6), 998-1004 (1985)
24. P. C. Chaumet and M. Nieto-Vesperinas, "Time averaged total force on a diplar sphere in an electromagnetic field," Opt. Lett. 25(15), 1065-1067 (2000)

25. B. T. Draine, "The discrete-dipole approximation and its application to interstellar graphite grains," Astrophys. J. 333(2), 848-872 (1988).

26. A. Ashkin, "Forces of a single-beam gradient laser trap on a dielectric sphere in the ray optics regime," Biophys. J. 61(2), 569-582 (1992).

27. L. Novotny and B. Hecht, Principles of Nano-Optics, Cambridge University Press, Cambridge, England (2006).

28. K. Svoboda and S. M. Block, "Optical trapping of metallic Rayleigh particles," Opt. Lett. 19(13), 930-932 (1994).

29. P. M. Hansen, V. K. Bhatia, N. Harrit, and L. Oddershede, "Expanding the optical trapping range of gold nanoparticles," Nano Lett. 5(10), 1937-1942 (2005)

30. Y. Seol, A. E. Carpenter, and T. T. Perkins, "Gold nanoparticles: enhanced optical trapping and sensitivity coupled with significant heating," Opt. Lett. 31(16), 2429-2431 (2006)

31. M. Dienerowitz, M. Mazilu, and K. Dholakia, "Optical manipulation of nanoparticles: a review," J. Nanophoton. 2, 021875 (2008).

32. A. Pendleton, S. Kundu, and H. Liang, "Controlled synthesis of titanium nanochains using a template," J. Nanopart. Res. 11, 505-510 (2009).

33. J. Q. Qin, X. L. Wang, D. Jia, J. Chen, Y. X. Fan, J. P. Ding, and H. T. Wang, "FDTD approach to optical forces of tightly focused vector beams on metal particles," Opt. Express 17(10), 8407-8416 (2009).

34. R. Saija, P. Denti, F. Borghese, O. M. Marago, and M. A. Iati, "Optical trapping calculations for metal nanoparticles: comparison with experimental data for $\mathrm{Au}$ and Ag spheres," Opt. Express 17(12), 10231-10241 (2009).

35. F. Hajizadeh and S. N. S. Reihani, "Optimized optical trapping of gold nanoparticles," Opt. Express 18(2), 551-559 (2010).

36. K. K. Jain, "Applications of nanobiotechnology in clinical diagnostics," Clin. Chem. 53(11), 2002-2009 (2007).

37. J. D. Jackson, Classical Electrodynamics, Wiley, Hoboken, NJ (1999).

38. J. P. Barton, "Internal and near-surface electromagnetic fields for a spheroidal particle with arbitrary illumination," Appl. Opt. 34(24), 5542-5551 (1995).

39. J. P. Barton, D. R. Alexander, and S. A. Schaub, "Theoretical determination of net radiation force and torque for a spherical particle illuminated by a focused laser beam," J. Appl. Phys. 66(10), 45944602 (1989)

40. A. Rohrbach, "Stiffness of optical traps: quantitative agreement between experiment and electromagnetic theory," Phys. Rev. Lett 95(16), 168102 (2005)

41. A. Rohrbach and E. H. Stelzer, "Trapping forces, force constants, and potential depths for dielectric spheres in the presence of spherical aberrations," Appl. Opt. 41(13), 2494-2507 (2002).

42. D. N. Moothoo, J. Arlt, R. S. Conroy, F. Akerboom, A. Voit, and K Dholakia, "Beth's experiment using optical tweezers," Am. J. Phys. 69(3), 271-276 (2001)

43. S. P. Smith, S. R. Bhalotra, A. L. Brody, B. L. Brown, E. K. Boyda, and M. Prentiss, "Inexpensive optical tweezers for undergraduate laboratories," Am. J. Phys. 67(1), 26-35 (1999).

44. W. M. Lee, P. J. Reece, R. F. Marchington, N. K. Metzger, and K. Dholakia, "Construction and calibration of an optical trap on a fluorescence optical microscope," Nat. Protoc. 2(12), 3226-3238 (2007).

45. S. M. Block, L. S. Goldstein, and B. J. Schnapp, "Bead movement by single kinesin molecules studied with optical tweezers," Nature 348(6299), 348-352 (1990).

46. B. J. Schnapp and T. S. Reese, "Dynein is the motor for retrograde axonal transport of organelles," Proc. Natl. Acad. Sci. U.S.A. 86(5), 1548-1552 (1989)

47. J. T. Finer, R. M. Simmons, and J. A. Spudich, "Single myosin molecule mechanics: piconewton forces and nanometre steps," Nature 368(6467), 113-119 (1994).

48. J. P. Rickgauer, D. N. Fuller, S. Grimes, P. J. Jardine, D. L. Anderson, and D. E. Smith, "Portal motor velocity and internal force resisting viral DNA packaging in bacteriophage phi29," Biophys. J. 94(1), 159-167 (2008).

49. E. A. Abbondanzieri, W. J. Greenleaf, J. W. Shaevitz, R. Landick, and S. M. Block, "Direct observation of base-pair stepping by RNA polymerase," Nature 438(7067), 460-465 (2005). 
50 J. D. Wen, L. Lancaster, C. Hodges, A. C. Zeri, S. H. Yoshimura, H. F. Noller, C. Bustamante, and I. Tinoco, "Following translation by single ribosomes one codon at a time," Nature 452(7187), 598-603 (2008).

51. C. Cecconi, E. A. Shank, C. Bustamante, and S. Marqusee, "Direct observation of the three-state folding of a single protein molecule," Science 309(5743), 2057-2060 (2005).

52. K. Berg-Sørensen and H. Flyvbjerg, "Power spectrum analysis for optical tweezers," Rev. Sci. Instrum. 75(3), 594-612 (2004).

53. K. M. Dohoney and J. Gelles, "[chi]-Sequence recognition and DNA translocation by single RecBCD helicase/nuclease molecules," $\mathrm{Na}$ ture 409(6818), 370-374 (2001).

54. H. Noji, R. Yasuda, M. Yoshida, and K. Kinosita, Jr., "Direct observation of the rotation of F1-ATPase," Nature 386(6622), 299-302 (1997).

55. R. S. Rock, S. E. Rice, A. L. Wells, T. J. Purcell, J. A. Spudich, and H. L. Sweeney, "Myosin VI is a processive motor with a large step size," Proc. Natl. Acad. Sci. U.S.A. 98(24), 13655-13659 (2001).

56. T. T. Perkins, "Optical traps for single molecule biophysics: a primer," Laser Photonics Rev. 3(1-2), 203-220 (2009).

57. M. D. Wang, M. J. Schnitzer, H. Yin, R. Landick, J. Gelles, and S. M. Block, "Force and velocity measured for single molecules of RNA polymerase," Science 282(5390), 902-907 (1998).

58. T. T. Perkins, H.-W. Li, R. V. Dalal, J. Gelles, and S. M. Block, "Forward and reverse motion of single RecBCD molecules on DNA," Biophys. J. 86(3), 1640-1648 (2004)

59. D. E. Smith, S. J. Tans, S. B. Smith, S. Grimes, D. L. Anderson, and C. Bustamante, "The bacteriophage straight phi29 portal motor can package DNA against a large internal force," Nature 413(6857), 748752 (2001).

60. T. T. Perkins, R. V. Dalal, P. G. Mitsis, and S. M. Block, "Sequencedependent pausing of single Lambda exonuclease molecules," Science 301(5641), 1914-1918 (2003).

61. H. Yin, M. D. Wang, K. Svoboda, R. Landick, S. M. Block, and J. Gelles, "Transcription against an applied force," Science 270(5242), 1653-1657 (1995)

62. B. van den Broek, M. A. Lomholt, S. M. Kalisch, R. Metzler, and G. J. Wuite, "How DNA coiling enhances target localization by proteins," Proc. Natl. Acad. Sci. U.S.A. 105(41), 15738-15742 (2008).

63. G. J. L. Wuite, S. B. Smith, M. Young, D. Keller, and C. Bustamante, "Single-molecule studies of the effect of template tension on T7 DNA polymerase activity," Nature 404(6773), 103-106 (2000).

64. S. J. Koch, A. Shundrovsky, B. C. Jantzen, and M. D. Wang, "Probing protein-DNA interactions by unzipping a single DNA double helix," Biophys. J. 83(2), 1098-1105 (2002).

65. D. S. Johnson, L. Bai, B. Y. Smith, S. S. Patel, and M. D. Wang, "Single-molecule studies reveal dynamics of DNA unwinding by the ring-shaped T7 helicase," Cell 129(7), 1299-1309 (2007).

66. B. D. Brower-Toland, C. L. Smith, R. C. Yeh, J. T. Lis, C. L. Peterson, and M. D. Wang, "Mechanical disruption of individual nucleosomes reveals a reversible multistage release of DNA," Proc. Natl. Acad. Sci. U.S.A. 99(4), 1960-1965 (2002).

67. M. L. Bennink, S. H. Leuba, G. H. Leno, J. Zlatanova, B. G. de Grooth, and J. Greve, "Unfolding individual nucleosomes by stretching single chromatin fibers with optical tweezers," Nat. Struct. Mol. Biol. 8(7), 606-610 (2001).

68. G. J. Gemmen, R. Millin, and D. E. Smith, "Tension-dependent DNA cleavage by restriction endonucleases: Two-site enzymes are 'switched off' at low force," Proc. Natl. Acad. Sci. U.S.A. 103(31), 11555-11560 (2006).

69. P. R. Bianco, L. R. Brewer, M. Corzett, R. Balhorn, Y. Yeh, S. C. Kowalczykowski, and R. J. Baskin, "Processive translocation and DNA unwinding by individual RecBCD enzyme molecules," Nature 409(6818), 374-378 (2001).

70. N. Handa, P. R. Bianco, R. J. Baskin, and S. C. Kowalczykowski, "Direct visualization of RecBCD movement reveals cotranslocation of the RecD motor after [chi] recognition," Mol. Cell 17(5), 745-750 (2005).

71. M. J. Lang, P. M. Fordyce, A. M. Engh, K. C. Neuman, and S. M. Block, "Simultaneous, coincident optical trapping and singlemolecule fluorescence," Nat. Methods 1(2), 133-139 (2004).

72. R. R. Brau, P. B. Tarsa, J. M. Ferrer, P. Lee, and M. J. Lang, "Interlaced optical force-fluorescence measurements for single molecule biophysics,” Biophys. J. 91(3), 1069-1077 (2006).
73. T. A. Waigh, "Microrheology of complex fluids," Rep. Prog. Phys. 68(3), 685-742 (2005).

74. K. C. Neuman and A. Nagy, "Single-molecule force spectroscopy: optical tweezers, magnetic tweezers and atomic force microscopy," Nat. Methods 5(6), 491-505 (2008).

75. C. Bustamante, Z. Bryant, and S. B. Smith, "Ten years of tension: single-molecule DNA mechanics," Nature 421(6921), 423-427 (2003).

76. J. R. Moffitt, Y. R. Chemla, S. B. Smith, and C. Bustamante, "Recent advances in optical tweezers," Аnпи. Rev. Biochem. 77(1), 205-228 (2008)

77. M. Capitanio, F. Vanzi, C. Broggio, R. Cicchi, D. Normanno, G. Romano, L. Sacconi, and F. S. Pavone, "Exploring molecular motors and switches at the single-molecule level," Microsc. Res. Tech. 65(45), 194-204 (2004).

78. R. D. Vale, B. J. Schnapp, T. Mitchison, E. Steuer, T. S. Reese, and M. P. Sheetz, "Different axoplasmic proteins generate movement in opposite directions along microtubules in vitro," Cell 43(3), 623-632 (1985).

79. D. J. Sharp, K. R. Yu, J. C. Sisson, W. Sullivan, and J. M. Scholey, "Antagonistic microtubule-sliding motors position mitotic centrosomes in drosophila early embryos," Nat. Cell Biol. 1(1), 51-54 (1999).

80. M. J. deCastro, R. M. Fondecave, L. A. Clarke, C. F. Schmidt, and R. J. Stewart, "Working strokes by single molecules of the kinesinrelated microtubule motor NCD," Nat. Cell Biol. 2(10), 724-729 (2000).

81. S. M. Block, "Kinesin motor mechanics: binding, stepping, tracking, gating, and limping," Biophys. J. 92(9), 2986-2995 (2007).

82. M. J. Lang and S. M. Block, "Resource letter: LBOT-1: laser-based optical tweezers," Am. J. Phys. 71(3), 201-215 (2003).

83. K. Svoboda, C. F. Schmidt, B. J. Schnapp, and S. M. Block, "Direct observation of kinesin stepping by optical trapping interferometry," Nature 365, 721-727 (1993)

84. H. Higuchi, E. Muto, Y. Inoue, and T. Yanagida, "Kinetics of force generation by single kinesin molecules activated by laser photolysis of caged ATP," Proc. Natl. Acad. Sci. U.S.A. 94(9), 4395-4400 (1997).

85. K. Svoboda, P. P. Mitra, and S. M. Block, "Fluctuation analysis of kinesin movement," Biophys. J. 68(4 Suppl), 69S (1995).

86. M. J. Schnitzer and S. M. Block, "Kinesin hydrolyses one ATP per 8 nm step," Nature 388(6640), 386-390 (1997).

87. S. M. Block, "Kinesin: what gives?," Cell 93(1), 5-8 (1998).

88. K. Svoboda and S. M. Block, "Force and velocity measured for single kinesin molecules," Cell 77(5), 773-784 (1994).

89. A. Ishijima, Y. Harada, H. Kojima, T. Funatsu, H. Higuchi, and T. Yanagida, "Single-molecule analysis of the actomyosin motor using nano-manipulation," Biochem. Biophys. Res. Commun. 199(2), 10571063 (1994).

90. J. E. Molloy, J. E. Burns, J. Kendrick-Jones, R. T. Tregear, and D. C. White, "Movement and force produced by a single myosin head," Nature 378(6553), 209-212 (1995).

91. J. E. Molloy, J. E. Burns, J. C. Sparrow, R. T. Tregear, J. KendrickJones, and D. C. White, "Single-molecule mechanics of heavy meromyosin and S1 interacting with rabbit or drosophila actins using optical tweezers," Biophys. J. 68(4 Suppl), 298S-303S, 303S-305S (1995).

92. M. E. Porter and W. S. Sale, "The $9+2$ axoneme anchors multiple inner arm dyneins and a network of kinases and phosphatases that control motility," J. Cell Biol. 151(5), 37-42 (2000).

93. C. Shingyoji, H. Higuchi, M. Yoshimura, E. Katayama, and T. Yanagida, "Dynein arms are oscillating force generators," Nature 393(6686), 711-714 (1998).

94. H. Hirakawa, and Y. Y. Toyoshima, "Processive movement of single $22 \mathrm{~S}$ dynein molecules occurs only at low ATP concentrations," Proc Natl. Acad. Sci. U.S.A. 97(6), 2533-2537 (2000)

95. A. Gennerich, A. P. Carter, S. L. Reck-Peterson, and R. D. Vale, "Force-induced bidirectional stepping of cytoplasmic dynein," Cell 131(5), 952-965 (2007).

96. D. Wendell, P. Jing, J. Geng, V. Subramaniam, T. J. Lee, C. Montemagno, and P. Guo, "Translocation of double-stranded DNA through membrane-adapted phi 29 motor protein nanopores," Nat. Nanotechnol. 4(11), 765-772 (2009).

97. M. Skurnik, M. Pajunen, and S. Kiljunen, "Biotechnological challenges of phage therapy," Biotechnol. Lett. 29(7), 995-1003 (2007). 
98. G. M. Cheetham, D. Jeruzalmi, and T. A. Steitz, "Structural basis for initiation of transcription from an RNA polymerase-promoter complex," Nature 399(6731), 80-83 (1999).

99. A. Ujvari and C. T. Martin, "Evidence for DNA bending at the T7 RNA polymerase promoter," J. Mol. Biol. 295(5), 1173-1184 (2000)

100. A. Sischka, C. Kleimann, W. Hachmann, M. M. Schafer, I. Seuffert, K. Tonsing, and D. Anselmetti, "Single beam optical tweezers setup with backscattered light detection for three-dimensional measurements on DNA and nanopores," Rev. Sci. Instrum. 79(6), 063702 (2008).

101. U. F. Keyser, J. v. d. Does, C. Dekker, and N. H. Dekker, "Optical tweezers for force measurements on DNA in nanopores," Rev. Sci. Instrum. 77(10), 105105 (2006)

102. U. F. Keyser, B. N. Koeleman, S. van Dorp, D. Krapf, R. M. M. Smeets, S. G. Lemay, N. H. Dekker, and C. Dekker, "Direct force measurements on DNA in a solid-state nanopore," Nat. Phys. 2(7), 473-477 (2006).

103. E. H. Trepagnier, A. Radenovic, D. Sivak, P. Geissler, and J. Liphardt, "Controlling DNA capture and propagation through artificial nanopores," Nano Lett. 7(9), 2824-2830 (2007).

104. M. Schroder and R. J. Kaufman, "The mammalian unfolded protein response," Annu. Rev. Biochem. 74, 739-789 (2005).

105. C. B. Anfinsen, E. Haber, M. Sela, and F. H. White, "The kinetics of formation of native ribonuclease during oxidation of the reduced polypeptide chain," Proc. Natl. Acad. Sci. U.S.A. 47(9), 1309-1314 (1961).

106. G. C. Spalding, J. Courtial, and R. Di Leonardo, "Holographic optical trapping," in Structured Light and Its Applications: An Introduction to Phase-Structured Beams and Nanoscale Optical Forces, D. L. Andrews, Ed., pp. 139-168, Elsevier, New York (2008).

107. K. Dholakia and W. M. Lee, "Optical trapping takes shape: the use of structured light fields," in Advances in Atomic, Molecular, and Optical Physics, pp. 261-337, Academic Press, London (2008).

108. M. Mazilu, D. J. Stevenson, F. Gunn-Moore, and K. Dholakia, "Light beats the spread: "non-diffracting" beams," Laser Photonics Rev. 4(4), 529-547 (2010).

109. J. E. Molloy and M. J. Padgett, "Lights, action: optical tweezers," Contemp. Phys. 43(4), 241-258 (2002).

110. H. Liang, K. T. Vu, P. Krishnan, T. C. Trang, D. Shin, S. Kimel, and M. W. Berns, "Wavelength dependence of cell cloning efficiency after optical trapping," Biophys. J. 70(3), 1529-1533 (1996).

111. E. Townes-Anderson, R. S. St Jules, D. M. Sherry, J. Lichtenberger, and M. Hassanain, "Micromanipulation of retinal neurons by optical tweezers," Mol. Vis 4, 12 (1998).

112. K. C. Neuman, E. H. Chadd, G. F. Liou, K. Bergman, and S. M. Block, "Characterization of photodamage to Escherichia coli in optical traps," Biophys. J. 77(5), 2856-2863 (1999).

113. M. B. Rasmussen, L. B. Oddershede, and H. Siegumfeldt, "Optical tweezers cause physiological damage to Escherichia coli and Listeria bacteria," Appl. Environ. Microbiol. 74(8), 2441-2446 (2008).

114. K. Sahu, S. Mohanty, and P. K. Gupta, "He-Ne laser $(632.8 \mathrm{~nm})$ pre-irradiation gives protection against DNA damage induced by a near-infrared trapping beam," J. Biophoton. 2(3), 140-144 (2009).

115. T. I. Karu, "Mitochondrial signaling in mammalian cells activated by red and near-IR radiation," Photochem. Photobiol. 84(5), 10911099 (2008).

116. G. Leitz, E. Fallman, S. Tuck, and O. Axner, "Stress response in Caenorhabditis elegans caused by optical tweezers: wavelength, power, and time dependence," Biophys. J. 82(4), 2224-2231 (2002).

117. G. D. Jeffries, J. S. Edgar, Y. Zhao, J. P. Shelby, C. Fong, and D. T. Chiu, "Using polarization-shaped optical vortex traps for single-cell nanosurgery," Arch. Hist. Exact Sci. 7(2), 415-420 (2007).

118. P. C. F. Møller and L. B. Oddershede, "Quantification of droplet deformation by electromagnetic trapping," Europhys. Lett. 88(4), 48005 (2009).

119. J. Guck, S. Schinkinger, B. Lincoln, F. Wottawah, S. Ebert, M. Romeyke, D. Lenz, H. M. Erickson, R. Ananthakrishnan, D. Mitchell, J. Kas, S. Ulvick, and C. Bilby, "Optical deformability as an inherent cell marker for testing malignant transformation and metastatic competence," Biophys. J. 88(5), 3689-3698 (2005).

120. F. Lautenschläger, S. Paschke, S. Schinkinger, A. Bruel, M. Beil, and J. Guck, "The regulatory role of cell mechanics for migration of differentiating myeloid cells," Proc. Natl. Acad. Sci. U.S.A. 106(37), 15696-15701 (2009).
121. T. W. Remmerbach, F. Wottawah, J. Dietrich, B. Lincoln, C. Wittekind, and J. Guck, "Oral cancer diagnosis by mechanical phenotyping," Cancer Res. 69(5), 1728-1732 (2009).

122. G. B. Liao, P. B. Bareil, Y. Sheng, and A. Chiou, "One-dimensional jumping optical tweezers for optical stretching of bi-concave human red blood cells," Opt. Express 16(3), 1996-2004 (2008).

123. P. J. Bronkhorst, G. J. Streekstra, J. Grimbergen, E. J. Nijhof, J. J. Sixma, and G. J. Brakenhoff, "A new method to study shape recovery of red blood cells using multiple optical trapping," Biophys. $J$. 69(5), 1666-1673 (1995).

124. J. Sleep, D. Wilson, R. Simmons, and W. Gratzer, "Elasticity of the red cell membrane and its relation to hemolytic disorders: an optical tweezers study," Biophys. J. 77(6), 3085-3095 (1999).

125. S. Suresh, J. Spatz, J. P. Mills, A. Micoulet, M. Dao, C. T. Lim, M. Beil, and T. Seufferlein, "Connections between single-cell biomechanics and human disease states: gastrointestinal cancer and malaria," Acta Biomater. 1(1), 15-30 (2005).

126. M. T. Wei, A. Zaorski, H. C. Yalcin, J. Wang, S. N. Ghadiali, A. Chiou, and H. D. Ou-Yang, "A comparative study of living cell micromechanical properties by oscillatory optical tweezers," Opt. Express 16(12), 8594-8603 (2008).

127. E. Eriksson, J. Enger, B. Nordlander, N. Erjavec, K. Ramser, M. Goksor, S. Hohmann, T. Nystrom, and D. Hanstorp, "A microfluidic system in combination with optical tweezers for analyzing rapid and reversible cytological alterations in single cells upon environmental changes," Lab Chip 7(1), 71-76 (2006)

128. C. T. A. Brown, D. J. Stevenson, X. Tsampoula, C. McDougall, A. A. Lagatsky, W. Sibbett, F. Gunn-Moore, and K. Dholakia, "Enhanced operation of femtosecond lasers and applications in cell transfection," J. Biophoton. 1(2), 183-199 (2008).

129. J. Baumgart, G. M. Hannapel, D. J. Stevenson, D. Day, M. Gu, and K. Dholakia, "Optical redistribution of microparticles and cells between microwells," Lab Chip 9, 1334-1336 (2009).

130. C. McDougall, D. J. Stevenson, C. T. A. Brown, F. Gunn-Moore, and K. Dholakia, "Targeted optical injection of gold nanoparticles into single mammalian cells," J. Biophoton. 2(12), 736-743 (2009).

131. B. Sun and D. T. Chiu, "Spatially and temporally resolved delivery of stimuli to single cells," J. Am. Chem. Soc. 125(13), 3702-3703 (2003).

132. B. Sun and D. T. Chiu, "Synthesis, loading, and application of individual nanocapsules for probing single-cell signaling," Langmuir 20(11), 4614-4620 (2004).

133. D. S. Campbell and C. E. Holt, "Apoptotic pathway and MAPKs differentially regulate chemotropic responses of retinal growth cones," Neuron 37(6), 939-952 (2003).

134. H. Kress, J.-G. Park, C. O. Mejean, J. D. Forster, J. Park, S. S. Walse, Y. Zhang, D. Wu, O. D. Weiner, T. M. Fahmy, and E. R. Dufresne, "Cell stimulation with optically manipulated microsources," Nat. Methods 6(12), 905-909 (2009).

135. S. Monneret, F. Belloni, and O. Soppera, "Combining fluidic reservoirs and optical tweezers to control beads/living cells contacts," Microfluid. Nanofluid. 3(6), 645-652 (2007).

136. A. Hoffmann, G. Meyer zu Hörste, G. Pilarczyk, S. Monajembashi, V. Uhl, and K. O. Greulich, "Optical tweezers for confocal microscopy," Appl. Phys. B 71(5), 747-753 (2000).

137. M. Goksör, J. Enger, and D. Hanstorp, "Optical manipulation in combination with multiphoton microscopy for single-cell studies," Appl. Opt. 43(25), 4831-4837 (2004).

138. S. Oddos, C. Dunsby, M. A. Purbhoo, A. Chauveau, D. M. Owen, M. A. Neil, D. M. Davis, and P. M. French, "High-speed highresolution imaging of intercellular immune synapses using optical tweezers," Biophys. J. 95(10), L66-L68 (2008).

139. K. Hayakawa, H. Tatsumi, and M. Sokabe, "Actin stress fibers transmit and focus force to activate mechanosensitive channels," $J$. Cell. Sci. 121(Pt 4), 496-503 (2008)

140. E. Eriksson, D. Engstrom, J. Scrimgeour, and M. Goksor, "Automated focusing of nuclei for time lapse experiments on single cells using holographic optical tweezers," Opt. Express 17(7), 5585-5594 (2009).

141. M. Lankers, J. Popp, and W. Kiefer, "Raman and fluorescence spectra of single optically trapped microdroplets in emulsions," Appl. Spectrosc. 48(9), 1166-1168 (1994).

142. C. Xie, M. A. Dinno, and Y. Q. Li, "Near-infrared Raman spectroscopy of single optically trapped biological cells," Opt. Lett. 27(4), 249-251 (2002). 
143. C. Xie, D. Chen, and Y. Q. Li, "Raman sorting and identification of single living micro-organisms with optical tweezers," Opt. Lett. 30(14), 1800-1802 (2005).

144. C. Creely, G. Volpe, G. Singh, M. Soler, and D. Petrov, "Raman imaging of floating cells," Opt. Express 13(16), 6105-6110 (2005).

145. F. Zheng, Y. Qin, and K. Chen, "Sensitivity map of laser tweezers Raman spectroscopy for single-cell analysis of colorectal cancer," $J$. Biomed. Opt. 12(3), 034002 (2007).

146. K. Chen, Y. Qin, F. Zheng, M. Sun, and D. Shi, "Diagnosis of colorectal cancer using Raman spectroscopy of laser-trapped single living epithelial cells," Opt. Lett. 31(13), 2015-2017 (2006).

147. T. J. Harvey, C. Hughes, A. D. Ward, E. C. Faria, A. Henderson, N. W. Clarke, M. D. Brown, R. D. Snook, and P. Gardner, "Classification of fixed urological cells using Raman tweezers," J. Biophoton. 2(1-2), 47-69 (2009).

148. E. Passegué, C. H. M. Jamieson, L. E. Ailles, and I. L. Weissman, "Normal and leukemic hematopoiesis: Are leukemias a stem cell disorder or a reacquisition of stem cell characteristics?," Proc. Natl. Acad. Sci. U.S.A. 100(Suppl 1), 11842-11849 (2003).

149. H. Brunner, A. Mayer, and H. Sussner, "Resonance Raman scattering on the haem group of oxy- and deoxyhaemoglobin," J. Mol. Biol. 70(1), 153-156 (1972).

150. K. Ramser, J. Enger, M. Goksor, D. Hanstorp, K. Logg, and M. Kall, "A microfluidic system enabling Raman measurements of the oxygenation cycle in single optically trapped red blood cells," Lab Chip 5(4), 431-436 (2005).

151. J. W. Chan, D. S. Taylor, S. M. Lane, T. Zwerdling, J. Tuscano, and T. Huser, "Nondestructive identification of individual leukemia cells by laser trapping raman spectroscopy," Anal. Chem. 80(6), 21802187 (2008).

152. A. C. De Luca, G. Rusciano, R. Ciancia, V. Martinelli, G. Pesce, B. Rotoli, L. Selvaggi, and A. Sasso, "Spectroscopical and mechanical characterization of normal and thalassemic red blood cells by Raman tweezers," Opt. Express 16(11), 7943-7957 (2008).

153. S. Rao, S. Balint, B. Cossins, V. Guallar, and D. Petrov, "Raman study of mechanically induced oxygenation state transition of red blood cells using optical tweezers," Biophys. J. 96(1), 209-216 (2009).

154. R. D. Snook, T. J. Harvey, E. C. Faria, and P. Gardner, "Raman tweezers and their application to the study of singly trapped eukaryotic cells," Integr. Biol. 1(1), 43-52 (2009).

155. J. Chan, S. Fore, S. Wachsmann-Hogiu, and T. Huser, "Raman spectroscopy and microscopy of individual cells and cellular components," Laser Photonics Rev. 2(5), 325-349 (2008).

156. D. V. Petrov, "Raman spectroscopy of optically trapped particles," J. Opt. A, Pure Appl. Opt. 9(8), S139-S156 (2007).

157. M. W. Berns, "Laser scissors and tweezers," Sci. Am. 278(4), 62-67 (1998).

158. R. W. Steubing, S. Cheng, W. H. Wright, Y. Numajiri, and M. W. Berns, "Laser induced cell fusion in combination with optical tweezers: the laser cell fusion trap," Cytometry 12(6), 505-510 (1991).

159. S. Seeger, S. Monajembashi, K. J. Hutter, G. Futterman, J. Wolfrum, and K. O. Greulich, "Application of laser optical tweezers in immunology and molecular genetics," Cytometry 12(6), 497-504 (1991).

160. S. Kumar, I. Z. Maxwell, A. Heisterkamp, T. R. Polte, T. P. Lele, M. Salanga, E. Mazur, and D. E. Ingber, "Viscoelastic retraction of single living stress fibers and its impact on cell shape, cytoskeletal organization, and extracellular matrix mechanics," Biophys. J. 90(10), 3762-3773 (2006).

161. H. Liang, W. H. Wright, C. L. Rieder, E. D. Salmon, G. Profeta, J. Andrews, Y. Liu, G. J. Sonek, and M. W. Berns, "Directed movement of chromosome arms and fragments in mitotic newt lung cells using optical scissors and optical tweezers," Exp. Cell Res. 213(1), 308-312 (1994).

162. M. W. Berns, Y. Tadir, H. Liang, and B. Tromberg, "Laser scissors and tweezers," Methods Cell Biol. 55, 71-98 (1998).

163. N. Ponelies, J. Scheef, A. Harim, G. Leitz, and K. O. Greulich, "Laser micromanipulators for biotechnology and genome research," J. Biotechnol. 35(2-3), 109-120 (1994).

164. K. Schütze, I. Becker, K.-F. Becker, S. Thalhammer, R. Stark, W. M. Heckl, M. Böhm, and H. Pösl, "Cut out or poke in--the key to the world of single genes: laser micromanipulation as a valuable tool on the look-out for the origin of disease," Genetic Anal. Biomol. Eng. 14(1), 1-8 (1997).
165. G. Leitz, C. Lundberg, E. Fällman, O. Axner, and A. Sellstedt, "Laser-based micromanipulation for separation and identification of individual Frankia vesicles," FEMS Microbiol. Lett. 224(1), 97-100 (2003).

166. D. J. Stevenson, F. J. Gunn-Moore, P. Campbell, and K. Dholakia, "Single cell optical transfection," J. R. Soc., Interface 7(47), 863871 (2009).

167. S. Kulin, R. Kishore, K. Helmerson, and L. Locascio, "Optical manipulation and fusion of liposomes as microreactors," Langmuir 19(20), 8206-8210 (2003).

168. D. Chiu, "Micro- and nano-scale chemical analysis of individual sub-cellular compartments," Trends Analyt. Chem. 22(9), 528-536 (2003).

169. D. T. Chiu, S. J. Lillard, R. H. Scheller, R. N. Zare, S. E. RodriguezCruz, E. R. Williams, O. Orwar, M. Sandberg, and J. A. Lundqvist, "Probing single secretory vesicles with capillary electrophoresis," Science 279(5354), 1190-1193 (1998).

170. S. S. Rubakhin, R. W. Garden, R. R. Fuller, and J. V. Sweedler, "Measuring the peptides in individual organelles with mass spectrometry," Nat. Biotechnol. 18(2), 172-175 (2000).

171. P. M. Lanigan, K. Chan, T. Ninkovic, R. H. Templer, P. M. French, A. J. de Mello, K. R. Willison, P. J. Parker, M. A. Neil, O. Ces, and D. R. Klug, "Spatially selective sampling of single cells using optically trapped fusogenic emulsion droplets: a new single-cell proteomic tool," J. R. Soc., Interface 5(Suppl 2), S161-168 (2008).

172. M. He, J. S. Edgar, G. D. M. Jeffries, R. M. Lorenz, J. P. Shelby, and D. T. Chiu, "Selective encapsulation of single cells and subcellular organelles into picoliter- and femtoliter-volume droplets," Anal. Chem. 77(6), 1539-1544 (2005).

173. A. Stromberg, A. Karlsson, F. Ryttsen, M. Davidson, D. T. Chiu, and O. Orwar, "Microfluidic device for combinatorial fusion of liposomes and cells," Anal. Chem. 73(1), 126-130 (2001).

174. A. Ashkin and J. M. Dziedzic, "Optical trapping and manipulation of viruses and bacteria," Science 235(4795), 1517-1520 (1987).

175. D. J. Odde and M. J. Renn, "Laser-guided direct writing of living cells," Biotechnol. Bioeng. 67(3), 312-318 (2000).

176. A. Tooker, E. Meng, J. Erickson, T. Yu-Chong, and J. Pine, "Biocomplatible parylene neurocages," IEEE Eng. Med. Biol. Mag. 24(6), 30-33 (2005).

177. J. Pine and G. Chow, "Moving live dissociated neurons with an optical tweezer," IEEE Trans. Biomed. Eng. 56(4), 1184-1188 (2009).

178. C. Luo, H. Li, C. Xiong, X. Peng, Q. Kou, Y. Chen, H. Ji, and Q. Ouyang, "The combination of optical tweezers and microwell array for cells physical manipulation and localization in microfluidic device," Biomed. Microdevices 9(4), 573-578 (2007).

179. G. M. Akselrod, W. Timp, U. Mirsaidov, Q. Zhao, C. Li, R. Timp, K. Timp, P. Matsudaira, and G. Timp, "Laser-guided assembly of heterotypic three-dimensional living cell microarrays," Biophys. J. 91(9), 3465-3473 (2006).

180. D. R. Burnham, G. D. Wright, N. D. Read, and D. McGloin, "Holographic and single beam optical manipulation of hyphal growth in filamentous fungi," J. Opt. A, Pure Appl. Opt. 9(8), S172-S179 (2007).

181. A. Ehrlicher, T. Betz, B. Stuhrmann, D. Koch, V. Milner, M. G. Raizen, and J. Kas, "Guiding neuronal growth with light," Proc. Natl. Acad. Sci. U.S.A. 99(25), 16024-16028 (2002).

182. D. J. Carnegie, D. J. Stevenson, F. Gunn-Moore, and K. Dholakia, "Guided neuronal growth using optical line traps," Opt. Express 16(14), 10507 (2008).

183. D. J. Carnegie, T. Cizmar, J. Baumgartl, F. J. Gunn-Moore, and K. Dholakia, "Automated laser guidance of neuronal growth cones using a spatial light modulator," J. Biophoton. 2(11), 682-692 (2009).

184. D. J. Stevenson, T. K. Lake, B. Agate, V. Gárcés-Chávez, K. Dholakia, and F. Gunn-Moore, "Optically guided neuronal growth at near infrared wavelengths," Opt. Express 14(21), 9786-9793 (2006).

185. A. Welle, S. Horn, J. Schimmelpfeng, and D. Kalka, "Photochemically patterned polymer surfaces for controlled PC-12 adhesion and neurite guidance," J. Neurosci. Methods 142(2), 243-250 (2005).

186. G. C. McKay, C. Henderson, E. Goldie, G. Connel, C. Westmoreland, and M. H. Grant, "Cryopreservation of rat hepatocyte monolayers: cell viability and cytochrome $\mathrm{P} 450$ content in post-thaw cultures," Toxicol. In Vitro 16(1), 71-79 (2002). 
187. A. A. Chen, A. M. Derfus, S. R. Khetani, and S. N. Bhatia, "Quantum dots to monitor RNAi delivery and improve gene silencing," Nucleic Acids Res. 33(22), e190 (2005).

188. S. R. Khetani, G. Szulgit, J. A. Del Rio, C. Barlow, and S. Bhatia, "Exploring interactions between rat hepatocytes and nonparenchymal cells using gene expression profiling," Hepatology 40(3), 545554 (2004).

189. Y. Nahmias, E. S. Robert, M. V. Catherine, and J. O. David, "Laserguided direct writing for three-dimensional tissue engineering," Biotechnol. Bioeng. 92(2), 129-136 (2005).

190. N. Arneborg, H. Siegumfeldt, G. Andersen, P. Nissen, R. V. Daria, P. J. Rodrigo, and J. Glückstad, "Interactive optical trapping shows that confinement is a determinant of growth in a mixed yeast culture," FEMS Microbiol. Lett. 245(1), 155-159 (2005).

191. M. J. Fulwyler, "Electronic separation of biological cells by volume," Science 150(698), 910-911 (1965).

192. M. M. Wang, E. Tu, D. E. Raymond, J. M. Yang, H. Zhang, N Hagen, B. Dees, E. M. Mercer, A. H. Forster, I. Kariv, P. J. Marchand, and W. F. Butler, "Microfluidic sorting of mammalian cells by optical force switching," Nat. Biotechnol. 23(1), 83-87 (2005).

193. K. Dholakia, W. M. Lee, L. Paterson, M. P. MacDonald, R. McDonald, I. Andreev, P. Mthunzi, C. T. A. Brown, R. F. Marchington, and A. C. Riches, "Optical separation of cells on potential energy landscapes: Enhancement with dielectric tagging," IEEE J. Sel. Top. Quantum Electron. 13(6), 1646-1654 (2007)

194. T. D. Perroud, J. N. Kaiser, J. C. Sy, T. W. Lane, C. S. Branda, A. K.
Singh, and K. D. Patel, "Microfluidic-based cell sorting of Francisella tularensis infected macrophages using optical forces," Anal. Chem. 80(16), 6365-6372 (2008).

195. K. Dholakia, M. P. MacDonald, P. Zemánek, and T. Cizmár, "Cellular and colloidal separation using optical forces," in Methods in Cell Biology, pp. 467-495, Academic Press, New York (2007).

196. L. Paterson, E. Papagiakoumou, G. Milne, V. Garces-Chavez, S. A. Tatarkova, W. Sibbett, F. J. Gunn-Moore, P. E. Bryant, A. C. Riches, and K. Dholakia, "Light-induced cell separation in a tailored optical landscape," Appl. Phys. Lett. 87(12), 123901 (2005).

197. L. Paterson, E. Papagiakoumou, G. Milne, V. Garces-Chavez, T. Briscoe, W. Sibbett, K. Dholakia, and A. C. Riches, "Passive optical separation within a "nondiffracting' light beam," J. Biomed. Opt. 12(5), 054017 (2007)

198. T. Imasaka, Y. Kawabata, T. Kaneta, and Y. Ishidzu, "Optical chromatography," Anal. Chem. 67(11), 1763-1765 (1995).

199. S. J. Hart, A. Terray, T. A. Leski, J. Arnold, and R. Stroud, "Discovery of a significant optical chromatographic difference between spores of Bacillus anthracis and its close relative, Bacillus thuringiensis," Anal. Chem. 78(9), 3221-3225 (2006).

200. S. Miki, T. Kaneta, and T. Imasaka, "Visualization of an immunological reaction between single antigen and antibody molecules by optical chromatography," Anal. Chim. Acta 404(1), 1-6 (2000).

201. T. Hatano, T. Kaneta, and T. Imasaka, "Application of optical chromatography to immunoassay," Anal. Chem. 69(14), 2711-2715 (1997). 